\title{
Light stop in the MSSM after LHC Run 1
}

\author{
Geneviève Bélanger, ${ }^{a}$ Diptimoy Ghosh, ${ }^{b, c}$ Rohini Godbole ${ }^{d}$ and Suchita Kulkarni ${ }^{e, f}$ \\ ${ }^{a}$ LAPTH, Université Savoie Mont Blanc, CNRS, \\ B.P.110, F-74941 Annecy-le-Vieux, France \\ ${ }^{b}$ INFN, Sezione di Roma, \\ Piazzale A. Moro 2, I-00185 Roma, Italy \\ ${ }^{c}$ Department of Particle Physics and Astrophysics, Weizmann Institute of Science, \\ Rehovot 76100, Israel \\ ${ }^{d}$ Center for High Energy Physics, Indian Institute of Science, \\ Bangalore, 560012, India \\ ${ }^{e}$ Laboratoire de Physique Subatomique et de Cosmologie, Université Grenoble-Alpes, CNRS/IN2P3, \\ 53 avenue des Martyrs, F-38026 Grenoble Cedex, France \\ ${ }^{f}$ Institut für Hochenergiephysik, Österreichische Akademie der Wissenschaften, \\ Nikolsdorfer Gasse 18, 1050 Wien, Austria \\ E-mail: genevieve.belanger@lapth.cnrs.fr, \\ diptimoy.ghosh@weizmann.ac.il, rohini@cts.iisc.ernet.in, \\ suchita.kulkarni@oeaw.ac.at
}

ABSTRACT: The discovery of a Higgs boson with a mass of $126 \mathrm{GeV}$ at the LHC when combined with the non-observation of new physics both in direct and indirect searches imposes strong constraints on supersymmetric models and in particular on the top squark sector. The experiments for direct detection of dark matter have provided with yet more constraints on the neutralino LSP mass and its interactions. After imposing limits from the Higgs, flavour and dark matter sectors, we examine the feasibility for a light stop in the context of the pMSSM, in light of current results for stop and other SUSY searches at the LHC. We only require that the neutralino dark matter explains a fraction of the cosmologically measured dark matter abundance. We find that a stop with mass below $\sim 500 \mathrm{GeV}$ is still allowed. We further study various probes of the light stop scenario that could be performed at the LHC Run-II either through direct searches for the light and heavy stop, or SUSY searches not currently available in simplified model results. Moreover we study the characteristics of heavy Higgs for the points in the parameter space allowed by all the available constraints and illustrate the region with large cross sections to fermionic or electroweakino channels. Finally we show that nearly all scenarios with a small stop-LSP mass difference will be tested by Xenon1T provided the NLSP is a chargino, thus probing a region hard to access at the LHC.

KEYWORDS: Supersymmetry Phenomenology

ARXIV EPRINT: 1506.00665 


\section{Contents}

1 Introduction $\quad 1$

2 Analysis set-up $\quad 4$

3 Indirect constraints 5

4 Direct search constraints $\quad 8$

5 Results 9

6 Probing light stop scenarios at the LHC 14

$\begin{array}{lll}6.1 & \text { Improving simplified models interpretations at } 8 \mathrm{TeV} & 14\end{array}$

$\begin{array}{lll}6.2 & \text { Stop NLSP at LHC } 14 \mathrm{TeV} & 19\end{array}$

$\begin{array}{lll}6.3 & \text { Decays of heavier stop } & 21\end{array}$

6.4 Decays of heavy Higgs 24

$\begin{array}{lll}7 & \text { Complementarity with dark matter searches } & 27\end{array}$

8 Conclusions $\quad 29$

\section{Introduction}

The Large Hadron Collider (LHC) has completed its first run (Run-I) with an unprecedented success. A Higgs particle has been discovered with a mass $\sim 126 \mathrm{GeV}$ [1-6]. Its couplings to the Standard Model (SM) electroweak gauge bosons have been established to be close to the SM expectations by measurements of signal rates [6-15] and spin-parity determinations [7, 16-18]. While there remains considerable room for deviations in the couplings to fermions, no sign of any New Physics (NP) has been detected yet. The search for new states near the electroweak scale has also been frustrating. The trudge of null results has shrunk the parameter spaces of weak scale NP models considerably, and models of weak scale Supersymmetry (SUSY) are not exceptions.

Indeed, the general tone at the moment is fairly lugubrious for SUSY enthusiasts. The simplest versions of the Minimal Supersymmetric Standard Model (MSSM), with the simplest assumptions about the high-scale theory, are under increasing tension with a wide range of experimental data including the $\sim 126 \mathrm{GeV}$ mass of the Higgs boson [19-22]. However, the above argument can be turned around to advocate many convincing reasons to study low energy SUSY. For example, that the electroweak symmetry is broken by an elementary scalar whole mass is below $135 \mathrm{GeV}$, is, in fact, a prediction of the MSSM. One should also be reminded that the MSSM has excellent decoupling properties which 
keep electroweak precision observables under control and to some extent also ameliorate the tension with limits on flavour observables. Moreover, the states which are directly related to the naturalness of Electro-Weak Symmetry Breaking (EWSB) are not constrained severely by the direct searches yet.

Existence of these states around the $\mathrm{TeV}$ scale is implied by demands of naturalness, which is a much discussed issue in the context of Supersymmetric theories (for example see $[23,24])$. The main point can be understood by considering the issue of stabilization of the Higgs mass against radiative corrections. To be specific, the correction to the Higgs mass due to radiative effects can be written as,

$$
\delta m_{h}^{2}\left(\Lambda_{\mathrm{EW}}\right) \sim \Lambda_{\mathrm{SUSY}}^{2} \ln \left(\frac{\Lambda_{\mathrm{mess}}}{\Lambda_{\mathrm{EW}}}\right),
$$

where $\Lambda_{\text {mess }}$ denotes the scale at which SUSY breaking effects are mediated to the MSSM and a common mass scale $\Lambda_{\text {SUSY }}$ for all the SUSY particles has been assumed. Eq. (1.1) immediately makes it clear why SUSY particles (especially those which couple strongly to the Higgs) $\lesssim \mathrm{TeV}$ are desired.

For moderate to large $\tan \beta \equiv\left\langle H_{u}\right\rangle /\left\langle H_{d}\right\rangle$, e.g. $\tan \beta \gtrsim 2$, the Higgs mass in the MSSM can be written as [23]

$$
m_{h}^{2}=-2\left(|\mu|^{2}+\left.m_{H_{u}}^{2}\right|_{\text {tree }}+\left.m_{H_{u}}^{2}\right|_{\text {rad }}\right),
$$

where $\mu$ is the supersymmetric Higgs mass parameter, and $\left.m_{H_{u}}^{2}\right|_{\text {tree }}$ and $\left.m_{H_{u}}^{2}\right|_{\text {rad }}$ are the tree-level and radiative contributions to the soft SUSY breaking mass squared for $H_{u}$. The dominant radiative correction to $m_{H_{u}}^{2}$ proportional to the top quark Yukawa coupling is given by [23],

$$
\left.m_{H_{u}}^{2}\right|_{\mathrm{rad}} \simeq-\frac{3 y_{t}^{2}}{8 \pi^{2}}\left(m_{\widetilde{Q}_{3}}^{2}+m_{\widetilde{U}_{3}}^{2}+\left|A_{t}\right|^{2}\right) \ln \left(\frac{\Lambda_{\mathrm{mess}}}{\mathrm{M}_{\tilde{\mathrm{t}}}}\right)
$$

where $y_{t}$ is the top Yukawa coupling, $m_{\widetilde{Q}_{3}}^{2}$ and $m_{\widetilde{U}_{3}}^{2}$ are the soft SUSY breaking mass squared parameters for the third-generation squark doublet and singlet up-type squark, $A_{t}$ is the scalar trilinear interaction parameter for the top squarks ${ }^{1}$ and $\mathrm{M}_{\tilde{\mathrm{t}}}$ denotes an average mass scale for the top squarks. Recall that in Supersummetric theories a light Higgs is 'natural' in the sense that the stabilization of the Higgs mass around the EW scale is guaranteed by the symmetry. The destabilizing effects come from SUSY breaking. If none of the terms on the right-hand-side of eq. (1.2) are much larger than the left-hand-side then it implies that no fine tuning of parameters in the theory is needed to guarantee the low Higgs mass. Thus the amount of 'cancellations' (fine tuning) required to satisfy eq. (1.2) is then a measure of 'naturalness'. As an example if we define the fine-tuning parameter $\Delta=\left.2 m_{H_{u}}^{2}\right|_{\mathrm{rad}} / m_{h}^{2}$ one gets an upper limit on the top squark mass scale as a function of the fine-tuning parameter [25-27],

$$
\widetilde{A_{t}} \equiv \sqrt{m_{\tilde{t}_{1}}^{2}+m_{\tilde{t}_{2}}^{2}+A_{t}^{2}} \lesssim 600 \mathrm{GeV} \sqrt{\frac{3}{\ln \left(\Lambda_{\text {mess }} / \mathrm{TeV}\right)}} \sqrt{\frac{\Delta}{5}} .
$$

\footnotetext{
${ }^{1}$ Note that we use stop and top squark interchangeably.
} 
Upper bounds on $\mu$ and the gluino mass can also be obtained in a similar way,

$$
\begin{aligned}
\mu & \lesssim 200 \mathrm{GeV} \sqrt{\frac{\Delta}{5}}, \\
M_{3} & \lesssim 900 \mathrm{GeV}\left(\frac{3}{\ln \left(\Lambda_{\text {mess }} / \mathrm{TeV}\right)}\right) \sqrt{\frac{\Delta}{5}} .
\end{aligned}
$$

Equation (1.6) follows from taking into account, in the leading-logarithm (LL) approximation, corrections to $m_{h}^{2}$ coming from gluino mass. These come from the gluino induced corrections to the Higgs potential arising at two loops which in turn come from gluino corrections to the stop mass at one loop.

If we forget about the Higgs mass and other indirect constraints for a moment, then from direct searches alone one can still have $m_{\widetilde{t}_{1}} \sim m_{\widetilde{t}_{2}} \sim 500 \mathrm{GeV}$ and $A_{t} \sim 0$. This amounts to a tuning $\Delta<10$ (assuming $\Lambda_{\text {mess }}=20 \mathrm{TeV}$ ). However, the direct search bound on the gluino mass $m_{\widetilde{g}} \gtrsim 1500 \mathrm{GeV}$ requires, according to eq. (1.6), the tuning to be $\Delta \gtrsim 15$. This means that, as far as the direct search bounds are concerned, it is the gluino mass that has stronger effect on fine tuning than the stop mass. Of course, when constraints from the Higgs mass are taken into account, low values for $A_{t}$ are not allowed (if at least one of the top squarks is desired to be light). This makes the tuning much worse $(\Delta>50)$.

The effect of gluino mass on fine-tuning can also be understood if one considers the running of the stop mass. Indeed the top squark is a scalar and its mass is subject to the same fine tuning problem as the Higgs mass. More precisely, the leading contribution is given by [23]

$$
\frac{d m_{\widetilde{t}}^{2}}{d \ln (\mu)}=-\frac{1}{16 \pi^{2}} \frac{32}{3} g_{3}^{2} M_{3}^{2}
$$

This means that the stop mass is attracted towards the gluino mass at low energies and thus a light stop does not seem very 'natural' in view of the rather high lower bounds on the gluino mass implied by LHC Run-I data. One way to allow a light stop and still be consistent with naturalness, is to somehow weaken the rather strong bounds on the gluino mass implied by the current LHC data. This can be done in SUSY models like compressed SUSY, stealth SUSY and R-parity violating SUSY, hence the renewed interest in such models [28-33].

One must further appreciate that the naturalness criteria, to some extent, tend to be subjective. In fact, ref. [34], argues that an appropriate model-independent measure of fine tuning is not in terms of the high scale quantities, but rather in terms of $\mu$. Thus a light Higgsino is the one robust demand one can make on the particle spectrum by requiring naturalness, without making any assumptions about the high scale physics. In this analysis they construct a measure of naturalness which is 'independent' of the precise model of the high scale physics. This measure can be small even when the conventional measures of fine tuning such as $\Delta$ take large values. Due to such subjectivity in the naturalness criteria, in our analysis we will cover values of parameters beyond the nominal upper bounds indicated by eqs. (1.4)-(1.6), for a given value of $\Delta$. 
Finally, irrespective of the amount of fine tuning introduced it is interesting to investigate to which extent light stop masses are allowed by all the existing direct and indirect measurements, and to see how well this region can be tested at the LHC. Here, we will work within the framework of the $\mathrm{CP}$ and flavour conserving phenomenological MSSM (pMSSM) with 19 free parameters [35, 36]. Within this model we will concentrate on the electroweak scale parameters that are most relevant for the Higgs and dark matter (DM) sector, that is the gaugino, Higgs and third generation squark parameters. We then explore the parameter space of the model allowed by flavour constraints, Higgs properties and Higgs searches, direct LHC searches for SUSY particles as well as DM constraints (relic density and direct detection).

The collider, flavour and DM constraints on the general pMSSM were explored in several publications [37-45] and the impact of stop searches was also considered [41, 43, 44, 46-56]. Possible probes to improve the bounds on stops and sbottoms with new observables and/or better background reduction have been explored in several works [57-73] and the difficult case of a compressed spectrum has been considered [74-84]. In this work we concentrate specifically on the parameter space of the model where a light stop is allowed and we rely on simplified model constraints on the stop mass at LHC Run-I. The aim is to understand the constraining power of the current search results and demonstrate possible ways in which these constraints can be improved. We incorporate recent LHC limits on SUSY particles using SModelS $[85,86]$ - a tool that exploits the simplified models results of the SUSY searches from the ATLAS and CMS collaborations. Finally, after characterizing the remaining parameter space, we discuss the different channels available to further probe light stop scenarios in the MSSM. These include LHC SUSY searches, the associated production of stops with light Higgs, the monojet search for degenerate stop-neutralino, searches for heavy Higgses as well as direct/indirect DM detection.

This paper is organized as follows. The set-up for the analysis is presented in section 2 followed by a summary of the indirect and direct constraints in section 3. Our results for the remaining available parameter space of the pMSSM with stops below $1.5 \mathrm{TeV}$ are discussed in section 5 together with the potential for further probing the model with various collider and astro-particle observables. Our conclusions are presented in section 6 .

\section{Analysis set-up}

Here we have chosen a simplified version of the pMSSM where only the ten parameters most relevant for the Higgs and DM sector are let to vary. All other parameters (squarks of the first and second generation and all sleptons) are fixed to a value large enough to evade all the LHC constraints. The ranges of the values of pMSSM parameters we consider are listed in table 1 . We performed a flat random scan for values of parameters in these ranges. Note that sleptons close in mass to the LSP can give an important contribution to DM (co-)annihilation, for example this occurs frequently for staus within the constrained MSSM. We ignore this possibility here since the importance of coannihilation will be illustrated with third generation squarks, moreover coannihilation with sleptons rely on 


\begin{tabular}{|l|c|}
\hline Parameter & Scan range \\
\hline U(1) gaugino mass parameter: $M_{1}$ & $20-2000$ \\
\hline SU(2) gaugino mass parameter: $M_{2}$ & $100-2000$ \\
\hline $\begin{array}{l}\text { Ratio of the vacuum expectation values of the two } \\
\text { Higgs doublets: } \tan \beta\end{array}$ & $2-55$ \\
\hline Higgsino mass parameter: $\mu$ & $100-3000$ \\
\hline Pseudo-scalar mass parameter: $m_{A}$ & $100-2000$ \\
\hline Stop tri-linear coupling: $A_{t}$ & $-5000-5000$ \\
\hline Sbottom tri-linear coupling: $A_{b}$ & $-5000-5000$ \\
\hline $\begin{array}{l}\text { Mass parameter for the left handed third generation } \\
\text { squark doublet: } m_{\widetilde{Q}_{3}}\end{array}$ & $100-2000$ \\
\hline Mass parameter for the right handed stop: $m_{\widetilde{U}_{3}}$ & $100-2000$ \\
\hline Mass parameter for the right handed sbottom: $m_{\widetilde{D}_{3}}$ & $100-2000$ \\
\hline
\end{tabular}

Table 1. Scan ranges for the pMSSM parameters, all dimension full parameters are in GeV. The values of all the slepton mass parameters as well as $m_{\widetilde{Q}_{1,2}}, m_{\widetilde{U}_{1,2}}, m_{\widetilde{D}_{1,2}}$ and $M_{3}$ are set to $2 \mathrm{TeV}$. All the $A$-terms other than $A_{t}$ and $A_{b}$ are assumed to vanish.

fine-tuning of parameters from unrelated sectors when no assumption is made about the underlying high scale model.

We have used SuSpect-2.41 [87] to compute the pMSSM mass spectrum for a given set of input parameters. This includes two-loop corrections to the Higgs mass and NLO corrections to SUSY particle masses. In the first step of our scan, we have generated $\sim 0.75$ Million pMSSM points for which the following statements apply,

- All the criteria in SuSpect-2.41 for a theoretically valid point are satisfied,

- The pMSSM spectrum has a neutralino LSP,

- The lightest CP even Higgs boson mass (as computed by SuSpect-2.41) $m_{h}$ satisfies $118 \mathrm{GeV}<m_{h}<130 \mathrm{GeV}$,

- The lightest stop and the LSP satisfy $m_{\widetilde{t}_{1}}<1500 \mathrm{GeV}$ and $m_{\widetilde{\chi}_{1}^{0}}<800 \mathrm{GeV}$ respectively.

Once the above step is done, we assess the impact of Higgs boson signal strengths, flavour violating observables, DM relic density and direct detection cross section, LEP data and finally, direct SUSY searches at the LHC on the selected set of $\sim 0.75$ Million pMSSM points.

Note that we have checked the vacuum stability and the absence of charge and colour breaking minima via SuSpect. A more refined analysis along the lines of [88-90] might improve the constraints in the large $A_{t}$ region.

\section{Indirect constraints}

- Higgs data: in order to study the compatibility of the pMSSM models with Higgs data we use HiggsBounds-4.1.0 [91, 92] and HiggsSignals-1.1.0 [93] which are linked 
to FeynHiggs for the computation of Higgs mass and signal strengths. As far as the theoretical uncertainty in the Higgs mass calculation is concerned, we use the estimate given by FeynHiggs-2.10.0 [94]. While calculating the $p$-value in HiggsSignals, we set the number of free model parameters $N_{p}=10$. The $p$-value is required to be more than 0.05 for an allowed parameter point. Note that the range for the Higgs mass assumed in our preselection will automatically be reduced by imposing these constraints.

- Flavour data: flavour physics has played a crucial role in the construction of the SM as well as constraining NP beyond the SM. The flavour structure of the SM is indeed very special, and any generic NP model suffers from large flavour violations in contradiction with the wealth of data from B-factories and recently also from $\mathrm{LHCb}$. In the pMSSM, because of the degeneracy of the first two squark generations the flavour violation involving the first two generation of fermions is mild. Moreover, as we have set the masses of the first two generation of squarks to $2 \mathrm{TeV}$ (which is allowed by direct searches at the LHC), their contributions decouple. Thus, the flavour constraints mainly arise from processes involving the third generation of quarks, for example, decays involving $b \rightarrow s$ transition. ${ }^{2}$ In particular, the flavour changing $B$ meson decays e.g., the radiative decay $\mathcal{B}\left(B_{d} \rightarrow X_{s} \gamma\right)$ and the fully leptonic decay $\mathcal{B}\left(B_{s} \rightarrow \mu^{+} \mu^{-}\right)$are known to place important constraints on the MSSM parameter space. In this work we have used the following limits for these branching ratios.

$$
\begin{aligned}
2.78 \times 10^{-4} \leq \mathcal{B}\left(B_{d} \rightarrow X_{s} \gamma\right) & \leq 4.08 \times 10^{-4}, \\
1.43 \times 10^{-9} \leq \mathcal{B}\left(B_{s} \rightarrow \mu^{+} \mu^{-}\right) & \leq 4.37 \times 10^{-9} .
\end{aligned}
$$

In addition, we have also imposed the following limits on the branching ratio of $B_{d} \rightarrow \mu^{+} \mu^{-}$,

$$
0.79 \times 10^{-10} \leq \mathcal{B}\left(B_{d} \rightarrow \mu^{+} \mu^{-}\right) \leq 6.80 \times 10^{-10} .
$$

In the numerical analysis, we have used the public code SuperIso-3.3 [103] to compute these branching ratios.

Note that we have not considered a few other potentially important observables such as the anomalous magnetic moment of the muon, $(g-2)_{\mu}$, the branching ratios of the two body leptonic decay of the $B$-meson $\mathcal{B}(B \rightarrow \tau \nu)$ and the three body semileptonic decay $\mathcal{B}\left(B \rightarrow D\left(D^{*}\right) \tau \nu\right)$. As far as $(g-2)_{\mu}$ is concerned, the SUSY contribution needs to be at least $1.0 \times 10^{-9}$ in order to be consistent with the measured value at the $2 \sigma$ level $[104,105]$. This requires the existence of light sleptons and electroweak gauginos which can be easily achieved in the pMSSM [53]. We set the slepton mass parameters to a high value for simplicity.

\footnotetext{
${ }^{2}$ Interestingly, the LHCb collaboration has reported hints of NP in some of the $B$ meson decay modes involving quark level $b \rightarrow s$ transitions [95, 96]. Although, there are pending issues with the reliability of the theoretical SM predictions and firm claims of the existence of NP can not be made yet, several NP explanations of these "deviations" have been proposed [97-101]. Unfortunately, a NP explanation within the MSSM seems unlikely [102].
} 
The leptonic decay $B \rightarrow \tau \nu$ which has tree level SUSY contribution from the charged Higgs exchange diagrams is also known to provide stringent constraints on the SUSY parameter space [106]. Interestingly, the most recent measurement by the Belle collaboration has brought down this branching ratio to a much smaller value than earlier measured [107], thus relaxing the tension with the SM prediction. All our points satisfy this constraint since many other constraints force the charged Higgs to be rather heavy any way.

Let us now discuss about the three body semileptonic decay $\mathcal{B}(B \rightarrow D \tau \nu)$ and $\mathcal{B}\left(B \rightarrow D^{*} \tau \nu\right)$. The BaBar collaboration measured the two quantities [108]

$$
R(D)=\frac{\mathcal{B}\left(\bar{B} \rightarrow D \tau^{-} \overline{\nu_{\tau}}\right)}{\mathcal{B}\left(\bar{B} \rightarrow D \ell^{-} \overline{\nu_{\ell}}\right)} \quad \text { and } \quad R\left(D^{*}\right)=\frac{\mathcal{B}\left(\bar{B} \rightarrow D^{*} \tau^{-} \overline{\nu_{\tau}}\right)}{\mathcal{B}\left(\bar{B} \rightarrow D^{*} \ell^{-} \overline{\nu_{\ell}}\right)}
$$

and reported a $3.4 \sigma$ deviation from the $\mathrm{SM}$ when the two measurements are taken together. This result motivated a number a phenomenological studies both in the context of specific models [109] as well as model independent approaches [110, 111]. The BaBar collaboration itself ruled out a Type-II Two Higgs Doublet Model (THDM) at $99.8 \%$ confidence level for any value of $\tan \beta / m_{H^{ \pm}}$based on this data. The same would apply to the Higgs sector of the MSSM which is also a Type-II THDM at the tree level. However, the existence of two neutrinos in the final state of these decays makes their measurements quite challenging experimentally and a confirmation of these results by another independent experiment (e.g., Belle II) is awaited.

- Dark matter relic density and direct detection: the DM relic density has been measured precisely by PLANCK, $\Omega h^{2}=0.1192 \pm 0.00024$ at $68 \%$ C.L. [112]. We impose the following upper bound

$$
\Omega h^{2} \leq 0.129
$$

which corresponds to the measured value after adding a $10 \%$ theoretical uncertainty - this number is a rough estimate of uncertainties that can arise for example from one-loop corrections to DM annihilation cross section [113-117]. ${ }^{3}$ We impose only the upper bound to allow for the possibility that the neutralino is only a fraction of the DM.

We also impose the mass dependent upper bound on the WIMP direct detection cross section obtained by the LUX experiment. For this, we have fitted the LUX upper bound [119] to an analytic formula which is given by,

$$
\begin{aligned}
\log _{10} \sigma_{\mathrm{SI}}^{\mathrm{LUX}}= & \frac{7.029}{\left(\log _{10} m_{\mathrm{WIMP}}\right)^{2}}-\frac{7.161}{\log _{10} m_{\mathrm{WIMP}}}-8.569 \\
& +0.755 \log _{10} m_{\mathrm{WIMP}}-0.003\left(\log _{10} m_{\mathrm{WIMP}}\right)^{2} .
\end{aligned}
$$

Based on this we apply the following constraint,

$$
\sigma_{\mathrm{SI}}<\xi \sigma_{\mathrm{SI}}^{\mathrm{LUX}}
$$

\footnotetext{
${ }^{3} \mathrm{~A}$ more precise value for the relic density has been released recently by PLANCK [118], this however has no impact on the results presented here since the theoretical uncertainty we assume is dominant.
} 
where

$$
\xi= \begin{cases}1 & \text { if } 0.1103<\Omega h^{2}<0.1289 \\ \frac{0.1196}{\Omega h^{2}} & \text { if } \Omega h^{2}<0.1103 .\end{cases}
$$

That is, if the relic density computed assuming the standard cosmological scenario falls below the PLANCK range, we consider that the neutralino constitutes only a fraction of the DM and explicitly ignore the possibility of regenerating DM although we will comment on this assumption in section 7. Moreover we do not make any assumptions about what would constitute the rest of the DM. Note that DM can also be searched for by indirect detection. In our analysis we have neither imposed the constraints from FermiLAT on photons [120], from PAMELA on antiprotons [121] as well as the preliminary limits from AMS on antiprotons [122], nor have we made any attempt to explain the anomalies observed by PAMELA [123] and AMS [124] on the positron spectrum. We do however, briefly discuss the impact of indirect searches in section 7. We use micrOMEGAs-3.5.5 [125] to calculate the DM relic density as well as the direct and indirect detection cross sections.

In the MSSM, it is well known that the composition of the neutralino is crucial for determining the DM properties. For neutralino annihilation to be efficient enough to have $\Omega h^{2} \leq 0.129$ requires either a LSP with a significant Higgsino or wino fraction or special tuning of parameters. In fact a dominantly Higgsino/wino LSP with a mass in the range $80 \mathrm{GeV}$ to $1-2 \mathrm{TeV}$ typically leads to $\Omega h^{2}<\Omega_{\text {PLANCK }} h^{2}$ because of efficient annihilation into $\mathrm{W}$ pairs. A mixed state with some bino component is therefore preferred. Direct detection cross section on the other hand is large for a mixed gaugino/Higgsino LSP, in particular for the one that leads to the exact range of the relic density determined by PLANCK. The only possibility for such mixed neutralino would be to lie above the TeV scale where the direct detection limits are weaker, we do not consider these masses since we want to highlight the SUSY spectrum below the TeV scale. Both cases with pure wino or pure Higgsino DM easily evade the direct detection constraints although the DM relic density cannot be entirely explained by neutralinos. Another possibility which allows also for a dominantly bino LSP consists in adjusting parameters such that $m_{\tilde{\chi}} \approx m_{Z} / 2$ or $m_{h} / 2$ or $m_{H} / 2$ thus providing a resonant enhancement of the cross section or having $m_{\tilde{\chi}} \approx m_{\text {sfermion. }}$. The contribution of coannihilation channels then reduces the relic density. The dominantly bino LSP is only allowed for light sfermions or when the mass is such that one can benefit from annihilation through a resonance in s-channel. Thus we expect to find a large number of scenarios with dominantly Higgsino or wino LSPs.

\section{Direct search constraints}

- LEP limits: the generic limits from LEP are obtained directly from micrOMEGAs and mainly exclude charged particles. The lower limit on chargino is $103 \mathrm{GeV}$ while those on sleptons, in particular staus are slightly weaker. In addition we have also imposed an upper limit on the $\mathrm{Z}$ invisible width, $\Gamma_{Z}<2 \mathrm{MeV}$ as well as constraints on neutralinos from $\sigma\left(e^{+} e^{-} \rightarrow \widetilde{\chi}_{1}^{0} \widetilde{\chi}_{i}^{0}\right)<0.1 \mathrm{pb}$ where the heavy neutralino, $\widetilde{\chi}_{i}^{0}$, decays mostly into hadrons and a LSP [126]. Such analyses constrain the very light 
neutralino LSP region, which is also strongly constrained by the upper bound on the DM relic density.

Finally we impose the condition that all particles decay promptly $(c \tau<0.05 \mathrm{~m})$. A strict requirement is that charged particles are not long-lived at the cosmological scale, we impose this more restrictive criteria because the LHC limits that we implement below assume prompt decays leading to Missing Transverse Energy (MET) in the final state.

- LHC limits: searches for SUSY at the LHC form an important ingredient to assess the viability of the scenario under consideration. Here, we describe our procedure for evaluating the LHC constraints. We use SModelS $[85,86]$, a tool designed to evaluate the LHC constraints on NP using simplified model spectra (SMS) results. SMODELS is designed to decompose the signal of any arbitrary NP spectrum with a $\mathbb{Z}_{2}$ symmetry into simplified model topologies and test it against the existing LHC bounds in the SMS context. The input to SModeLS can either be an SLHA file [127] containing the SUSY production cross sections $\sigma$ and the branching ratios for the SUSY decays, $\mathcal{B}$ or a LHE file [128]. For this work we used the SLHA input containing $\sigma$ and $\mathcal{B}$. The format for writing the production cross sections is specified in [129]. The production cross sections are computed using Pythia6.4.27 [130] and NLL-fast2.1 [131-138]. Given the information on the $\sigma$ and $\mathcal{B}$, SModelS computes $\sigma \times \mathcal{B}$ for each possible decay of SUSY particles. The information of relevance to check the results against the LHC limits is the mass vector of the SUSY particles, the SM decay products and the $\sigma \times \mathcal{B}$ of the resulting topologies. A topology resulting from such SLHA decomposition is considered if the $\sigma \times \mathcal{B}>\sigma_{\text {cut }}$, with $\sigma_{\text {cut }}$ set to $0.01 \mathrm{fb}$.

When dealing with an arbitrary NP spectrum, care must be taken to identify regions of compressed spectra as the decay products in such cases are not detected. SMODELS ignores such soft decays when the mass gap between the mother and the daughter particles is less than the user defined minimum mass gap, here we take $5 \mathrm{GeV}$. The resulting $\sigma \times \mathcal{B}$ for various SMS topologies were tested against ATLAS SMS interpretation for searches [139-151] and CMS searches [152-164].

\section{Results}

We start our discussion with table 2 where the impact of the various experimental data on the pMSSM parameter space is shown. Since we are randomly scanning over 10 uncorrelated parameters, it is important to generate enough points to populate all dimensions. The table shows the effect of each successive experimental constraint on a well populated flat scan over 10 dimensional parameter space. The number of pMSSM points which survive after each successive constraint is presented in the second column. Although no statistical meaning can be attached to these numbers, table 2 and the accompanying discussion helps to get an understanding of how the various type of data constrain the MSSM parameter space. It can be seen that out of the $\sim$ three quarter of a million points that were generated, only $60 \%$ of them successfully satisfy the observed Higgs boson mass, signal strength 


\begin{tabular}{|l|c|}
\hline Constraints & No. of models \\
\hline $\begin{array}{l}\text { Theory }+ \text { neutral LSP }+ \\
118 \mathrm{GeV}<m_{h}<130 \mathrm{GeV}+m_{\widetilde{t}_{1}}<1500 \mathrm{GeV}+m_{\widetilde{\chi}_{1}^{0}}<800 \mathrm{GeV}\end{array}$ & 741605 \\
\hline \multicolumn{2}{|c|}{ Indirect bounds } \\
\hline+ HiggsBounds + HiggsSignals & 435021 \\
\hline$+\mathcal{B}\left(B_{d} \rightarrow X_{s} \gamma\right)$ & 211313 \\
\hline$+\mathcal{B}\left(B_{s, d} \rightarrow \mu^{+} \mu^{-}\right)$ & 177961 \\
\hline$+\Omega h_{<}^{2}+\sigma_{\mathrm{SI}}^{\mathrm{LUX}}$ & 111167 \\
\hline \multicolumn{2}{|c|}{ Direct bounds } \\
\hline+ LEP & 59425 \\
\hline+ Long-lived chargino & 30754 \\
\hline+ LHC & 29266 \\
\hline
\end{tabular}

Table 2. Number of surviving pMSSM models after each cut.

data and other Higgs boson searches (which are implemented in HiggsBounds-4.1.0 and HiggsSignals-1.1.0). The severe impact of the measured branching ratio of $B_{d} \rightarrow X_{s} \gamma$ is also clear from the table. Out of all the models which satisfied Higgs data, only about $50 \%$ survive after the consistency with $B_{d} \rightarrow X_{s} \gamma$ branching ratio is imposed. It is worth mentioning that there is some amount of tension between the Higgs mass and the branching ratio of $B_{d} \rightarrow X_{s} \gamma$ in the MSSM. The latter gets contributions from chargino loops as well as charged Higgs loops. As the charged Higgs loop interferes constructively with the SM, its contribution is always positive. The chargino contribution on the other hand can be both constructive or destructive. For example, the stop-Higgsino contribution to the amplitude is proportional to $m_{t}^{2} \mu A_{t} \tan \beta / m_{\widetilde{t}}^{4}$ and hence, this contribution is enhanced for a large $A_{t}$. On the other hand, consistency with the measured value of the Higgs mass requires a large $A_{t}$ if a light $\widetilde{t}_{1}$ is desired.

The DM constraints also reduce significantly the number of allowed points. In particular when the LSP is lighter than the $\mathrm{W}$ the only allowed points are near $m_{Z} / 2$ or $m_{h} / 2$ corresponding to the annihilation through a $\mathrm{Z}$ or Higgs resonance. For heavier LSP masses the relic density upper bound basically selects LSP with large Higgsino or wino LSP, barring special configurations where coannihilations are important. Furthermore the mixed bino(wino)/Higgsino can be in conflict with the LUX direct detection bound as mentioned above. We therefore expect that the LSP will most of the time be dominantly Higgsino or wino which in both cases implies that it is almost degenerate with the chargino. Such degenerate chargino-neutralino lead to long lived charginos, leading to charged tracks at the LHC. As explained before, these scenarios are not considered in the present study and hence nearly half the points are discarded. Similarly the LEP limits on charged particles also rule out nearly half of the allowed points. Finally, LHC limits from the $8 \mathrm{TeV}$ run rule out about $5 \%$ of the allowed points. Below we discuss in more details the impact of LHC constraints from SUSY searches as obtained with SMODELS.

In figure 1 we plot the allowed and excluded points after applying SModELS. In the left panel we plot the excluded points on top of the allowed points while in the right panel, 

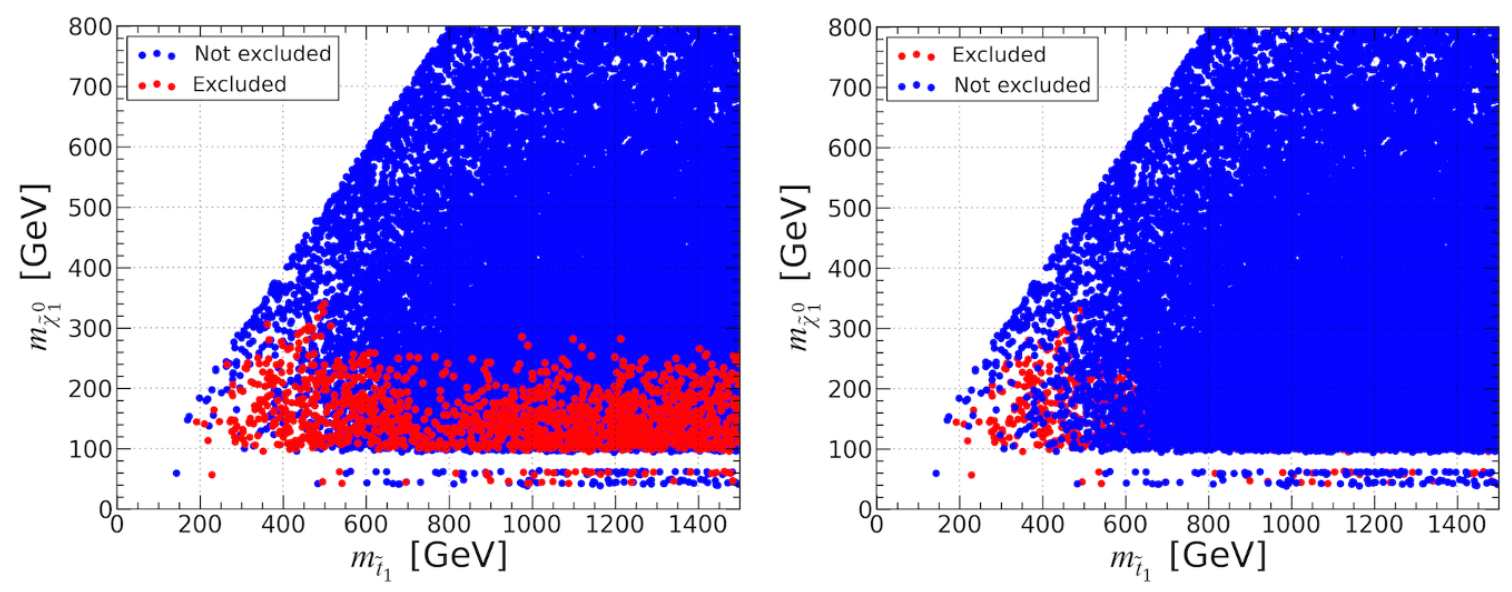

Figure 1. Summary of the allowed and excluded points by SModelS. In the left panel, the excluded points are displayed on top of the allowed points while in the right panel the allowed points are on top of the excluded points.

the plotting order is inverted. Clearly many points with light stop masses are not excluded by SMS results as implemented in SMODELS-1.0.3. It is possible to exclude many points up to the maximum stop mass considered $(1500 \mathrm{GeV})$. However, no SMS result has reach for LSP masses greater than $300 \mathrm{GeV}$ and therefore the region with higher LSP masses remains unconstrained.

The right panel shows that different configurations of the MSSM spectra can evade the SMS results, thus allowing very light stop masses — even below $200 \mathrm{GeV}$. Despite the fact that SMODELS combines topologies with the same final states and similar mass vectors, the main reason for the allowed region is that the SMS results obtained by LHC collaborations and used by SMODELS assume a $100 \%$ branching ratio for the decay under consideration while in the MSSM branching ratios are often below 100\%. For example consider a point in our scan with $m_{\widetilde{t}_{1}} \approx 380 \mathrm{GeV}$ and the rest of the spectra too heavy to contribute to limit setting. The dominant branching ratio for the stop is into $b \tilde{\chi}^{+}(77 \%)$, however this channel cannot be exploited as the chargino decays into a virtual $\mathrm{W}$ and the LSP, a channel which is not implemented in SModelS. Therefore only the channel $t \tilde{\chi}_{1}^{0}$ (with a $\mathrm{BR} \approx 0.13$ ) can be used to constrain the stop. This point gives $\sigma \times \mathcal{B} \approx 8.29 \mathrm{fb}$, whereas the experimental upper limit in CMS-SUS-13-011 derived assuming a $100 \% \mathcal{B}$ is $438 \mathrm{fb}$. Hence this point does not get excluded by SModeLS. The right panel of figure 1 thus justifies the possibility that a light stop is consistent with the current LHC SUSY searches as well as the observed Higgs properties and the heavy Higgs searches, along with the DM direct detection limits.

Figure 2 shows the breakdown of the most constraining analysis for the excluded points in the stop-LSP mass plane and chargino-LSP plane. For each of the excluded points, we select the most constraining analysis. ${ }^{4}$ It is possible that a point is excluded by more than one searches at the LHC. We overlay the exclusion lines obtained by ATLAS and CMS from

\footnotetext{
${ }^{4}$ The most constraining analysis is defined as the analysis which leads to the largest ratio of the theory cross section to the experimental upper limit.
} 

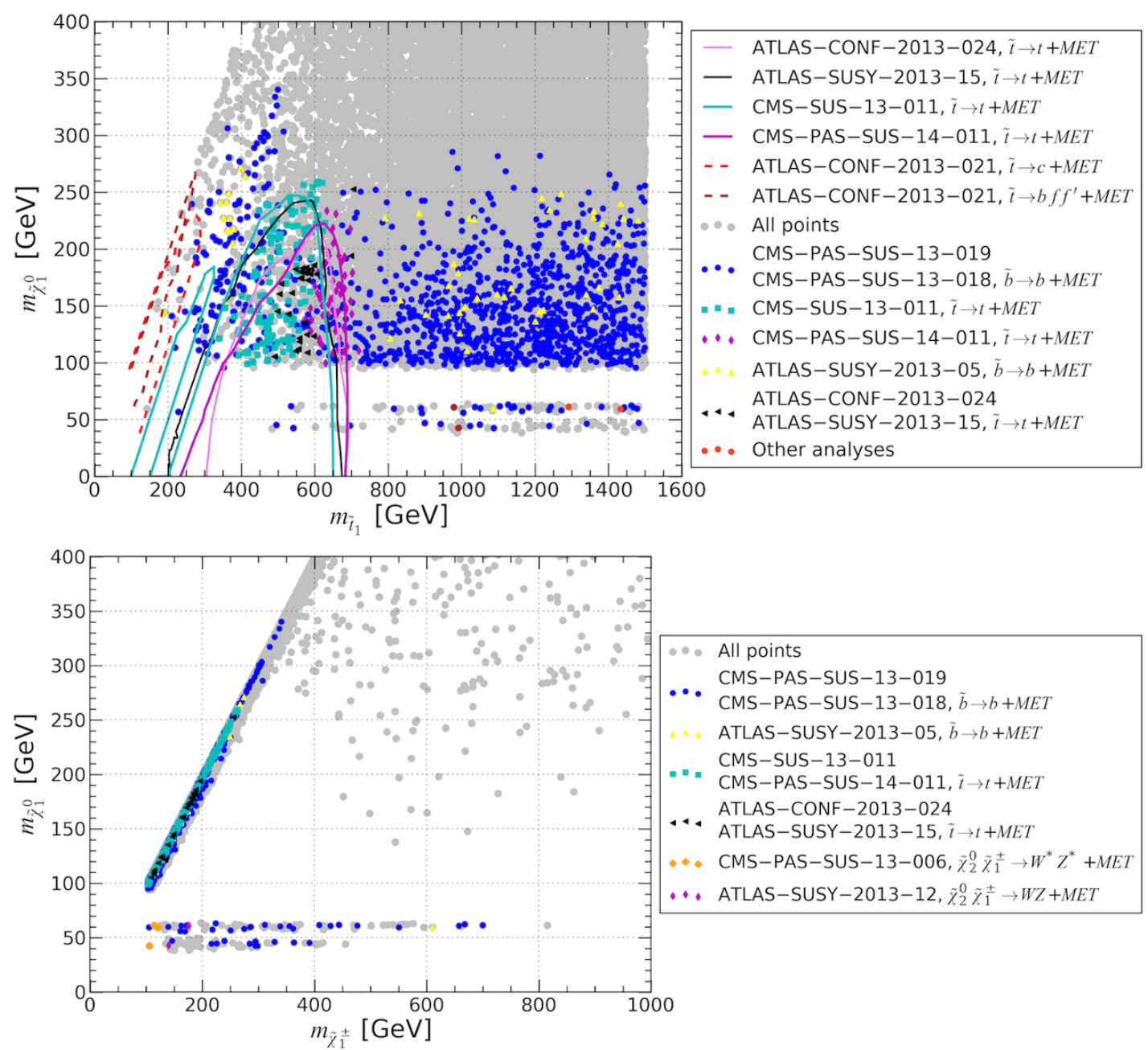

Figure 2. Breakdown of the most constraining analysis for the points excluded by SMODELS in the $m_{\widetilde{t}_{1}}-m_{\tilde{\chi}_{1}^{0}}$ mass plane (upper panel) and in the $m_{\widetilde{\chi}_{1}^{ \pm}}-m_{\tilde{\chi}_{1}^{0}}$ mass plane (lower panel). For comparison, exclusion curves from various ATLAS and CMS stop searches are overlaid (solid lines). The dashed line shows exclusions arising from ATLAS searche for stop decays to $c \widetilde{\chi}_{1}^{0}$ and to $b \widetilde{\chi}_{1}^{0} f f^{\prime}$.

stop searches to guide the eye. Indeed we see that most of the points excluded by the $\tilde{t} \rightarrow$ $t \widetilde{\chi}_{1}^{0}$ searches fall within the corresponding exclusion contours while higher stop masses are in fact excluded by constraints coming from sbottom searches. There are also significant constraints arising from sbottom searches for the light stop masses in the regime where $\tilde{t} \rightarrow t \widetilde{\chi}_{1}^{0}$ is kinematically forbidden while $\tilde{b} \rightarrow b \widetilde{\chi}_{1}^{0}$ is allowed. This kinematic configuration is common when $\widetilde{t}_{1}, \widetilde{b}_{1}$ are left-handed, thus sbottom searches provide indirect constraint on the light stop scenarios otherwise elusive at the LHC. For the kinematic edge where $m_{\tilde{t}}-m_{\tilde{\chi}_{1}^{0}}<$ $m_{t}$, four body decays of stops as well as decays via $c \widetilde{\chi}_{1}^{0}$ are also utilized at the LHC. Results from ATLAS [165] (for four body stop decay as well as decay via charm) and CMS [166] (stop decays via charm) searches are available. The ATLAS search yields stronger limits. Unfortunately, the cross section 95\% C.L. observed upper limit map on the $\sigma \times \mathcal{B}$ is not available in [165]. This search hence could not be included in SMODELS and was not used 

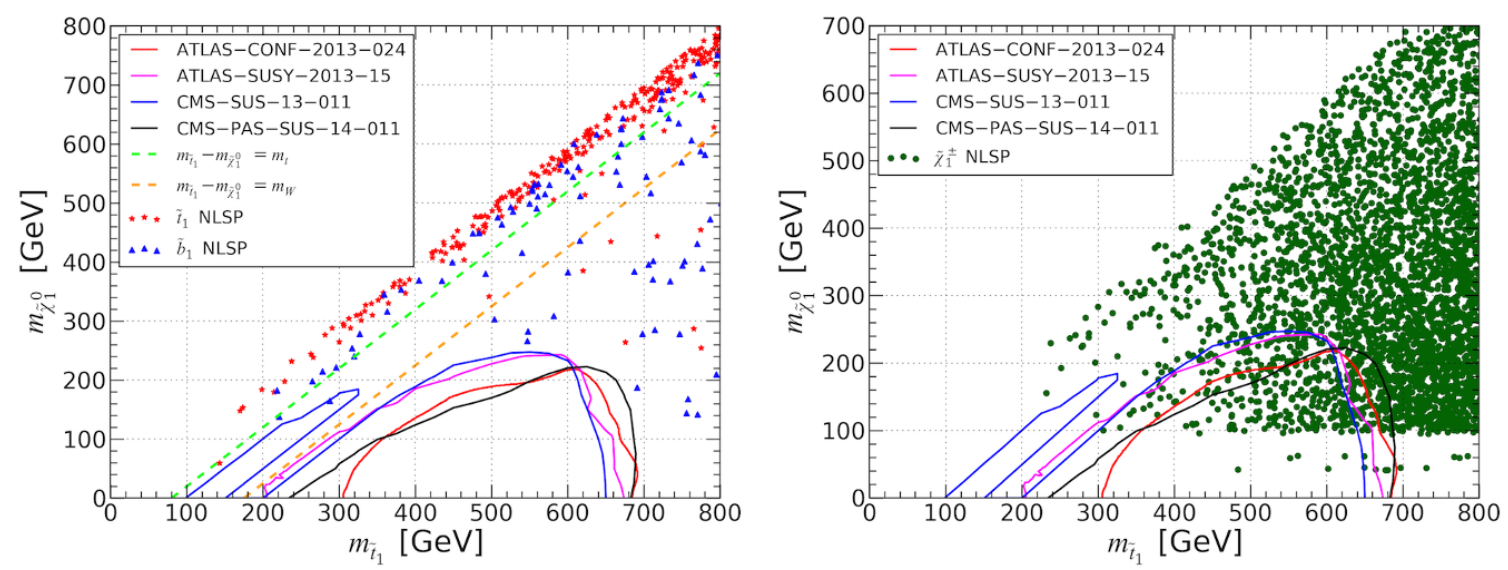

Figure 3. Allowed points in the $m_{\widetilde{t}_{1}}-m_{\widetilde{\chi}_{1}^{0}}$ plane when the NLSP is $m_{\widetilde{t}_{1}}$ or $m_{\widetilde{b}_{1}}$ (left panel), $m_{\widetilde{\chi}_{1}^{ \pm}}$ (right panel). The exclusions curves from the analyses of the stop searches used in testing the points in the $m_{\widetilde{t}_{1}}-m_{\widetilde{\chi}_{1}^{0}}$ plane. The lines with different colors and styles correspond to different SMS results for direct stop pair production decaying to $t \bar{t}+$ MET.

in this study. The red dashed exclusion lines obtained from the ATLAS searches [165] are overlaid in figure 2 (upper panel) for comparison. It can be seen that they do not cover a large region of parameter space, hence do not affect our conclusions drastically.

Figure 2 (lower panel) shows the exclusions in the $\widetilde{\chi}_{1}^{ \pm}-\widetilde{\chi}_{1}^{0}$ plane, three distinct branches can be seen. The points along $m_{\widetilde{\chi}_{1}^{0}} \approx 45 \mathrm{GeV}$ or $m_{\widetilde{\chi}_{1}^{0}} \approx 60 \mathrm{GeV}$ correspond to mostly binolike $\widetilde{\chi}_{1}^{0}$, while the points along the diagonal represent dominantly Higgsino or wino $\widetilde{\chi}_{1}^{0}$. The figure illustrates that most of the times, the chargino is nearly mass degenerate with the LSP and either decays invisibly or via an off-shell $W$ to LSP, thus evading the SMS limits which do not include either of these channels. The current searches for $\widetilde{\chi}_{2}^{0} \widetilde{\chi}_{1}^{ \pm}$decaying to WZ + MET therefore exclude only a few points in this parameter space. These are located along the bino branches of the plot. Furthermore, along the diagonal lines where the chargino decay is invisible, the searches for stop and sbottom pair production with direct decays to LSP or Next-to-Lightest Supersymmetric Particle (NLSP) contribute the most to the exclusion.

In figure 3 we plot the allowed points in the $m_{\widetilde{t}_{1}}-m_{\widetilde{\chi}_{1}^{0}}$ plane. We separate the total number of allowed points in three categories i) pMSSM points which have the lightest stop as the NSLP (red stars) ii) lightest sbottom as the NLSP (blue triangles) iii) lightest chargino as the NLSP (green circles). We have shown these three set of points separately to emphasize that most of the allowed parameter points where $\widetilde{t}_{1}$ is the NLSP (the left panel in figure 3) lie close to the $m_{\widetilde{t}_{1}}=m_{\widetilde{\chi}_{1}^{0}}$ line. The requirements on the relic density leading to the stop co-annihilation region is responsible for this strip. In these kind of scenarios, it is difficult to constrain these points via direct stop searches. The right panel shows that most of the points belong to the category of chargino NSLP as argued above. In such cases, the stop has cascade decays via chargino or heavier neutralinos, thus reducing the branching ratio for each mode and leading to weaker exclusions than expected from SMS results. Moreover only results where the chargino decays through a real $\mathrm{W}$ are included in SmodelS. 
We have demonstrated the exclusions obtained with the help of SMS results. However, it might be possible to obtain stronger exclusions by means of recasting an analysis, for example using the approach in [167-169]. This is clearly beyond the scope of this study. It is worth noting that allowing for light sleptons in the scan might lead to further exclusions driven by chargino-neutralino decays via intermediate sleptons. Furthermore, there are SMS interpretations for the decays of heavier stop e.g. stop searches with final states involving Higgs or $\mathrm{Z}$ boson [170]. As will be demonstrated in section 6.3 due to the constraint on the Higgs mass the $\widetilde{t}_{2}$ is always heavier than $\sim 700 \mathrm{GeV}$, where these searches currently do not have sensitivity.

\section{Probing light stop scenarios at the LHC}

From the results just presented it should be clear that the light stop scenarios in the pMSSM offer a variety of signatures at colliders. Note that this includes not only those from direct stop production but also from other light super particles. In particular, a light left-handed stop means, quite often, a light left-handed sbottom as well. Thus, in this section we first investigate the main signatures that could not be constrained by SModelS and suggest additional topologies which may be pursued at LHC14. Here we concentrate on final states produced by stop and sbottom decays and show missing topologies with large cross-section. We further also present missing topologies that arise from the electroweak sector for our allowed MSSM points. Then we examine other potential signatures from light stop associated production with an extra jet or a Higgs. Furthermore, we examine possible final states resulting from the decay of the heavier stop and we also discuss aspects of Heavy Higgs phenomenology for the set of MSSM points which are allowed in our light stop scenario.

\subsection{Improving simplified models interpretations at $8 \mathrm{TeV}$}

Figure 1 demonstrates the existence of a large number of points not excluded by SModeLS. In order to understand the characteristics of these points and to suggest further ways to constrain the non-excluded regions of parameter space, it is interesting to ask which SMS topologies not covered by current SMS searches in the SMODELS database prevail in these points. These are dubbed 'missing topologies' and will be discussed in this section in details. The missing topologies are derived purely on the basis of cross section times branching ratio $(\sigma \times \mathcal{B})$ computations and hence do not take into account the sensitivity of the experimental searches. For example, in the results that follow, the hadronic decays of $\mathrm{W}$ will show up most of the times because the branching ratio for $\mathrm{W}$ decays to quarks is higher than into leptons, however, it is more difficult to beat the backgrounds while searching for hadronic decays. Note that it is possible to constrain some of the missing topologies by means of reinterpreting the existing experimental searches, thus missing topologies are not always associated with a new signature.

The procedure used to derive missing topologies in SMODELS is as follows. Each SUSY point leads to more than one missing topology. SModeLS sums over the $\sigma \times \mathcal{B}$ for all the topologies irrespective of the mass vector of the SUSY particles. Up to 10 such topologies 

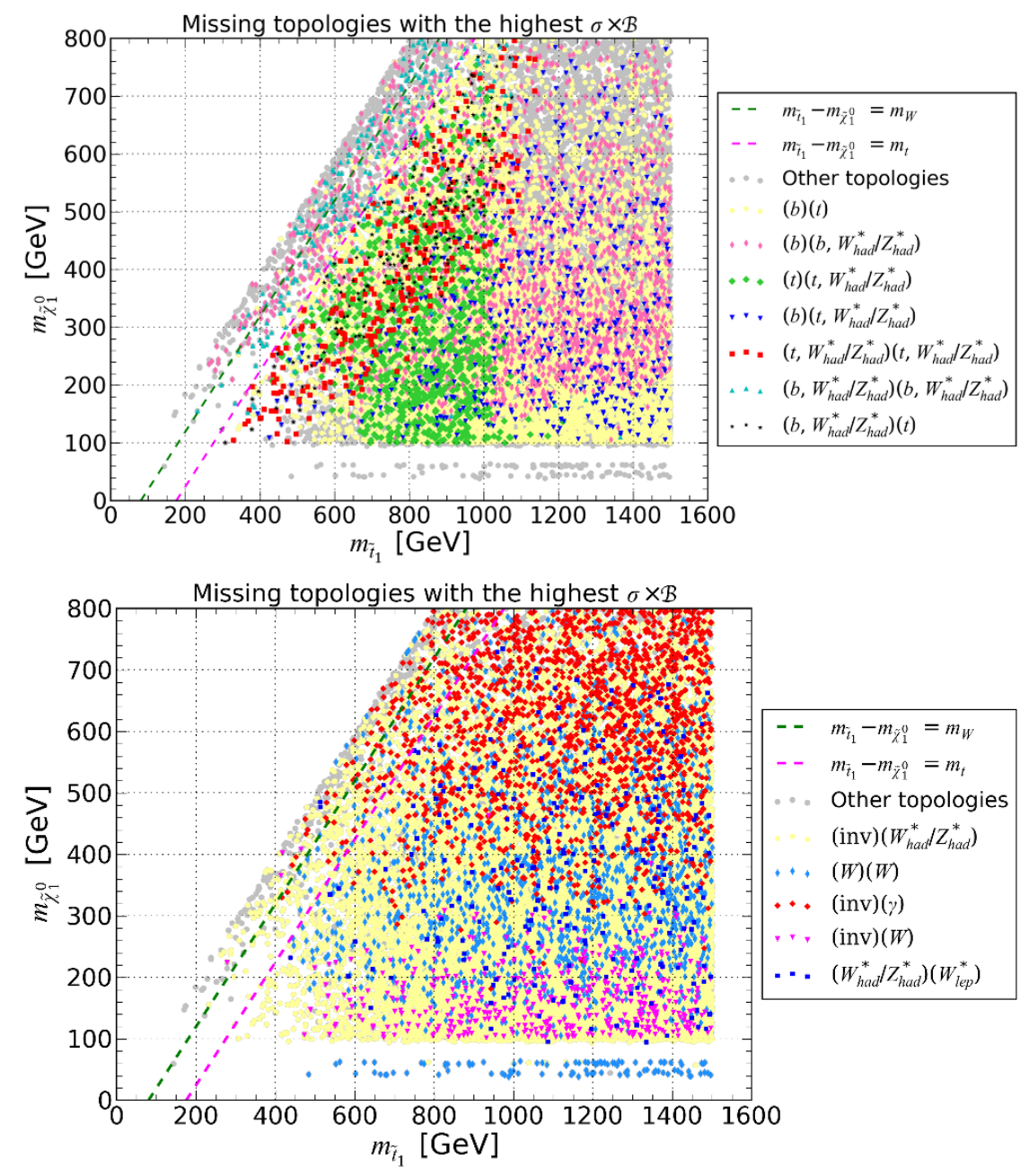

Figure 4. Most dominant missing topologies in the stop-LSP mass plane. For each non-excluded point, the SMS topology leading to the highest $\sigma \times \mathcal{B}$ is chosen. The seven most frequently occurring topologies are plotted, the frequency of topologies indicated in the legend are ordered (top to bottom) with the exception of the label 'other topologies'. The top panel shows the seven most frequently occurring topologies originating from stop/sbottom decays while the lower panel represents those originating from electroweakino decays.

with the highest $\sigma \times \mathcal{B}$ are recorded in the output. In the following results, we suppress pair production of LSP which occurs as a missing topology. Moreover, we do not consider any initial/final state radiation effects.

In figure 4, for each non-excluded point, the SMS topology leading to the highest $\sigma \times \mathcal{B}$ is chosen. We plot the seven most frequently occurring topologies originating from decays of stop/sbottom (upper panel) or electroweakinos (lower panel). The labels of the plot are written in a simplified notation with respect to the one used in SMODELS [85, 86], however it is easy to map the current notation to the original one. In this work, every branch is enclosed in parenthesis () and every vertex is separated with a comma (,). In both notations, it is assumed that every branch is accompanied with MET at the end of 
the cascade. The possible origins of these topologies are explained in table 3. Note that with this notation we do not distinguish particles and antiparticles and a sum over light quarks and light leptons is understood. In principle, decays apart from those illustrated in the table can contribute to the missing topologies however, because SMODELS does not keep track of the SUSY particles but only of their masses, this information is lost in the process of decomposition. Let us first concentrate on the upper panel of figure 4 . The most frequently occurring topology is $(b),(t)$, meaning $b+t+$ MET. The topology occurs via asymmetric decays of pair produced stop (sbottom) with one of the stops (sbottoms) decaying directly to top (bottom) + LSP while another stop (sbottom) decays to bottom (top) via chargino. The decay of chargino itself is invisible when the chargino-LSP mass gap is less than $5 \mathrm{GeV}$. Given the extreme degeneracy of the chargino-LSP masses the frequency of this topology is hardly a surprise. In general, this topology is difficult to be constrained by the current LHC searches as it leads to $2 \mathrm{~b}$ jets + one lepton + MET or $2 \mathrm{~b}$ jets +2 jets + MET final state. This is a topology with low jet multiplicity while most of the current searches for stops (sbottoms) require high multiplicity of light jets. Recently, limits for this mixed topology were made available by both the ATLAS [171] and CMS [172] collaborations. These limits assume exactly $50 \%$ branching ratios for each decay mode. The limits thus obtained are a sum over symmetric and asymmetric decay modes and do not represent upper limits on $\mathrm{b}+\mathrm{t}+\mathrm{MET}$ final state alone. For this reason, these limits are not applicable to our topology.

Other topologies involve the cascade decay of one or both pair-produced squarks into a quark and a heavier neutralino (chargino) which then decays into a LSP and an offshell $Z(W)$. It is important to notice that the topologies $(t)\left(t, W_{\text {had }}^{*} / Z_{\text {had }}^{*}\right.$ ), ( $t$, $\left.W_{\text {had }}^{*} / Z_{\text {had }}^{*}\right)\left(t, W_{\text {had }}^{*} / Z_{\text {had }}^{*}\right)$, (b, $\left.W_{\text {had }}^{*} / Z_{\text {had }}^{*}\right)(\mathrm{t})$ for which stops contribute, are suppressed for $m_{\widetilde{t}_{1}}>1 \mathrm{TeV}$. This is simply because the production cross section of stops heavier than $1 \mathrm{TeV}$ is extremely small. Thus the only relevant topologies are those resulting from sbottom decay, it occurs only when the right handed sbottom is lighter than the stops. Note that when $W_{\text {had }}^{*}$ or $Z_{\text {had }}^{*}$ are found in a missing topology, there is also the same topology with $W_{\text {lep }}^{*}$ or $Z_{\text {lep }}^{*}$ with a cross section reduced by the relative leptonic to hadronic branching ratio of the gauge boson. Despite the smaller cross section the leptonic final states typically have a much better signal to background ratio.

The most frequent topology resulting from electroweakino production and decays represented in the lower panel of figure 4 corresponds to associated production of the LSP with a chargino (heavier neutralino) decaying via an off-shell $W(Z)$. Other topologies get their dominant contributions from the decays of at least one heavier neutralino or chargino, as detailed in table 3. The very mixed nature of these electroweakino lead to a sizeable production cross sections despite heavier masses.

The main topologies, mono-W ( (inv) $(W)$ ), mono-photon ( (inv) $(\gamma))$ and diboson $((W)(W))^{5}$ cover different mass range $s$ for the LSP and this independently of the mass

\footnotetext{
${ }^{5}$ In principle, SMS results for this topology are available by ATLAS [143]. However, this result is not yet included in SMOdELS. The reach of this analysis at the moment is very limited, excluding chargino mass up to $180 \mathrm{GeV}$ and reaching up to maximum neutralino mass of $30 \mathrm{GeV}$. Inclusion of this search does not exclude any points as the LSP is always heavier than $\sim 40 \mathrm{GeV}$ for our scenario.
} 


\begin{tabular}{|c|c|}
\hline Topology & Decay \\
\hline (b) $\left(\mathrm{b}, W_{\text {had }}^{*} / Z_{\text {had }}^{*}\right)$ & $\widetilde{b}_{1} \widetilde{b}_{1} \rightarrow b \widetilde{\chi}_{1}^{0} b \widetilde{\chi}_{2}^{0} \rightarrow b \widetilde{\chi}_{1}^{0} b Z_{\mathrm{had}}^{*} \widetilde{\chi}_{1}^{0}$ \\
\hline (b) (t) & $\begin{array}{l}\widetilde{t}_{1} \widetilde{t}_{1} \rightarrow t \widetilde{\chi}_{1}^{0} b W_{\text {soft }} \widetilde{\chi}_{1}^{0} \\
\widetilde{b}_{1} \widetilde{b}_{1} \rightarrow b \widetilde{\chi}_{1}^{0} t W_{\text {soft }} \widetilde{\chi}_{1}^{0}\end{array}$ \\
\hline$\left(\mathrm{b}, W_{\text {had }}^{*} / Z_{\text {had }}^{*}\right)\left(\mathrm{b}, W_{\text {had }}^{*} / Z_{\text {had }}^{*}\right)$ & $\widetilde{t}_{1} \widetilde{t}_{1} \rightarrow b W_{\text {had }}^{*} \widetilde{\chi}_{1}^{0} b W_{\text {had }}^{*} \widetilde{\chi}_{1}^{0}$ \\
\hline$(\mathrm{t})\left(\mathrm{t}, W_{\mathrm{had}}^{*} / Z_{\mathrm{had}}^{*}\right)$ & $\begin{array}{l}\widetilde{t}_{1} \widetilde{t}_{1} \rightarrow t \widetilde{\chi}_{1}^{0} t \widetilde{\chi}_{2}^{0} \rightarrow t \widetilde{\chi}_{1}^{0} t Z_{\text {had }}^{*} \widetilde{\chi}_{1}^{0} \\
\widetilde{t}_{1} \widetilde{t}_{1} \rightarrow t \widetilde{\chi}_{2}^{0} t \widetilde{\chi}_{2}^{0} \rightarrow t Z_{\text {had }}^{*} \widetilde{\chi}_{1}^{0} t Z_{\text {had }}^{*} \widetilde{\chi}_{1}^{0}\end{array}$ \\
\hline (b) $\left(\mathrm{t}, W_{\text {had }}^{*} / Z_{\text {had }}^{*}\right)$ & $\begin{array}{l}\widetilde{t}_{1} \widetilde{t}_{1} \rightarrow t \widetilde{\chi}_{1}^{0} t \widetilde{\chi}_{2}^{0} \rightarrow t \widetilde{\chi}_{1}^{0} t Z_{\mathrm{had}}^{*} \widetilde{\chi}_{1}^{0} \\
\widetilde{t}_{1} \widetilde{t}_{1} \rightarrow t \widetilde{\chi}_{2}^{0} t \widetilde{\chi}_{2}^{0} \rightarrow t Z_{\nu \nu}^{*} \widetilde{\chi}_{1}^{0} t Z_{\mathrm{had}}^{*} \widetilde{\chi}_{1}^{0}\end{array}$ \\
\hline$\left(\mathrm{t}, W_{\text {had }}^{*} / Z_{\text {had }}^{*}\right)\left(\mathrm{t}, W_{\text {had }}^{*} / Z_{\text {had }}^{*}\right)$ & $\begin{array}{l}\widetilde{t}_{1} \widetilde{t}_{1} \rightarrow t \widetilde{\chi}_{2}^{0} t \widetilde{\chi}_{2}^{0} \rightarrow t Z_{\text {had }}^{*} \widetilde{\chi}_{1}^{0} t Z_{\text {had }}^{*} \widetilde{\chi}_{1}^{0} \\
\widetilde{b}_{1} \widetilde{b}_{1} \rightarrow t \widetilde{\chi}_{1}^{ \pm} t \widetilde{\chi}_{1}^{ \pm} \rightarrow t W_{\text {had }}^{*} \widetilde{\chi}_{1}^{0} t W_{\text {had }}^{*} \widetilde{\chi}_{1}^{0}\end{array}$ \\
\hline$\left(\mathrm{b}, W_{\text {had }}^{*} / Z_{\text {had }}^{*}\right)(\mathrm{t})$ & $\begin{array}{l}\widetilde{b}_{1} \widetilde{b}_{1} \rightarrow b \widetilde{\chi}_{2}^{0} t \widetilde{\chi}_{1}^{ \pm} \rightarrow b Z_{\text {had }}^{*} \widetilde{\chi}_{1}^{0} t W_{\text {soft }} \widetilde{\chi}_{1}^{0} \\
\widetilde{t}_{1} \widetilde{t}_{1} \rightarrow b \widetilde{\chi}_{1}^{ \pm} t \widetilde{\chi}_{1}^{0} \rightarrow b W_{\text {had }} \widetilde{\chi}_{1}^{0} t \widetilde{\chi}_{1}^{0}\end{array}$ \\
\hline (b) $\left(\mathrm{b}, Z_{\mathrm{lep}}^{*}\right)$ & $\widetilde{b}_{1} \widetilde{b}_{1} \rightarrow b \widetilde{\chi}_{1}^{0} b \widetilde{\chi}_{2}^{0} \rightarrow b \widetilde{\chi}_{1}^{0} b Z_{\mathrm{lep}}^{*} \widetilde{\chi}_{1}^{0}$ \\
\hline$(\mathrm{b})(\mathrm{b}, \gamma)$ & $\begin{array}{l}\widetilde{b}_{1} \widetilde{b}_{1} \rightarrow b \widetilde{\chi}_{1}^{0} b \widetilde{\chi}_{2}^{0} \rightarrow b \widetilde{\chi}_{1}^{0} b \gamma \widetilde{\chi}_{1}^{0} \\
\widetilde{b}_{1} \widetilde{b}_{1} \rightarrow b \widetilde{\chi}_{2}^{0} b \widetilde{\chi}_{2}^{0} \rightarrow b Z_{\nu \nu}^{*} \widetilde{\chi}_{1}^{0} b \gamma \widetilde{\chi}_{1}^{0}\end{array}$ \\
\hline$($ inv $)\left(W_{\text {had }}^{*} / Z_{\text {had }}^{*}\right)$ & $\begin{array}{l}\widetilde{\chi}_{1}^{ \pm} \widetilde{\chi}_{1}^{0} \rightarrow W_{\text {had }}^{*} \widetilde{\chi}_{1}^{0} \widetilde{\chi}_{1}^{0} \\
\widetilde{\chi}_{j}^{0} \widetilde{\chi}_{1}^{0} \rightarrow Z_{\text {had }}^{*} \widetilde{\chi}_{1}^{0} \widetilde{\chi}_{1}^{0}\end{array}$ \\
\hline$(\mathrm{W})(\mathrm{W})$ & $\tilde{\chi}_{2}^{ \pm} \widetilde{\chi}_{j}^{0} \rightarrow W \widetilde{\chi}_{1}^{0} W \widetilde{\chi}_{1}^{ \pm} \rightarrow W, \tilde{\chi}_{1}^{0} W W_{\text {soft }} \widetilde{\chi}_{1}^{0}$ \\
\hline (inv)(photon) & $\widetilde{\chi}_{1}^{ \pm} \widetilde{\chi}_{2}^{0} \rightarrow W_{\text {soft }} \widetilde{\chi}_{1}^{0} \gamma \widetilde{\chi}_{1}^{0}$ \\
\hline$(\mathrm{inv})(\mathrm{W})$ & $\widetilde{\chi}_{1}^{ \pm} \widetilde{\chi}_{3}^{0} \rightarrow W_{\text {soft }} \widetilde{\chi}_{1}^{0} W \widetilde{\chi}_{1}^{ \pm} \rightarrow W_{\text {soft }} \widetilde{\chi}_{1}^{0} W W_{\text {soft }} \widetilde{\chi}_{1}^{0}$ \\
\hline$(\operatorname{inv})\left(W_{\text {lep }}^{*}\right)$ & $\tilde{\chi}_{1}^{ \pm} \widetilde{\chi}_{1}^{0} \rightarrow W_{\mathrm{lep}}^{*} \widetilde{\chi}_{1}^{0} \widetilde{\chi}_{1}^{0}$ \\
\hline$(\mathrm{inv})\left(Z_{\text {lep }}^{*}\right)$ & $\tilde{\chi}_{1}^{ \pm} \widetilde{\chi}_{2}^{0} \rightarrow W_{\text {soft }} \widetilde{\chi}_{1}^{0} Z_{\text {lep }}^{*} \widetilde{\chi}_{1}^{0}$ \\
\hline$\left(W_{\text {had }}^{*} / Z_{\text {had }}^{*}\right)\left(W_{\text {lep }}^{*}\right)$ & $\begin{array}{l}\widetilde{\chi}_{2}^{0} \widetilde{\chi}_{1}^{ \pm} \rightarrow Z_{\text {had }}^{*} \widetilde{\chi}_{1}^{0} W_{\text {lep }}^{*} \widetilde{\chi}_{1}^{0} \\
\tilde{\chi}_{1}^{ \pm} \widetilde{\chi}_{1}^{ \pm} \rightarrow W_{\text {had }}^{*} \widetilde{\chi}_{1}^{0} W_{\text {lep }}^{*} \widetilde{\chi}_{1}^{0}\end{array}$ \\
\hline$($ inv $)(b, b)$ & $\widetilde{\chi}_{2}^{0} \widetilde{\chi}_{1}^{ \pm} \rightarrow Z_{b b}^{*} \widetilde{\chi}_{1}^{0} W_{\text {soft }} \widetilde{\chi}_{1}^{0}$ \\
\hline
\end{tabular}

Table 3. Missing topologies represented in figures 4, 5 and 6, written in SMODELS notation and the corresponding physical process. $W_{\text {soft }}$ represents soft decays of $\mathrm{W}$, which are undetected. $Z_{\text {lep }}^{*}$ and $W_{\text {lep }}^{*}$ represent the sum over all three generations of leptons, $Z_{\text {had }}^{*}$ and $W_{\text {had }}^{*}$ represent the sum over first two generation quarks.

of the stop. Mono-W topologies could be used to probe the region $100<m_{\widetilde{\chi}_{1}^{0}}<200 \mathrm{GeV}$ while the mono-photon dominates for higher LSP masses. Note that the mono-photon topology is here associated with $\widetilde{\chi}_{1}^{ \pm}+\widetilde{\chi}_{2}^{0}$ production where the chargino decays invisibly and the heavier neutralino decays via a loop-induced decay into the LSP and a photon. Because of the small difference between the $\widetilde{\chi}_{2}^{0}-\widetilde{\chi}_{1}^{0}$ masses, the loop-induced decay can have a large enough branching ratio (typically $\mathcal{O}(5 \%)$ ) to give a signature while the products of the three-body decay are too soft to be detected and are thus registered as a pure missing energy signature. Note that these photons could have a large $p_{T}$. The diboson topology which arises from heavier chargino decays cover the full mass range, in particular the bino- 


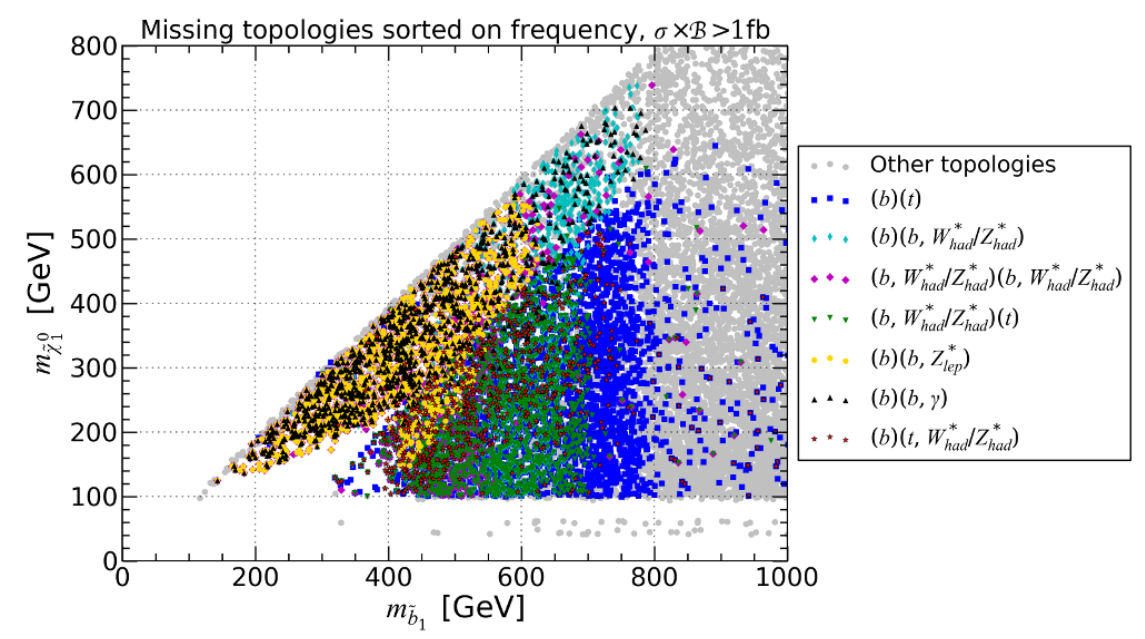

Figure 5. The figure shows missing topologies with cross section greater than $1 \mathrm{fb}$ originating from stop or sbottom decays and sorted on their frequency of occurrence. The seven most frequently occurring topologies are illustrated. Topologies originating from electroweakino decays are suppressed.

like neutralino branches (with $m_{\widetilde{\chi}_{1}^{0}} \approx 60 \mathrm{GeV}$ ) where there are no topologies occurring from the decays of stops/sbottoms. Updates of searches like [143] in the diboson final state from Run-II will thus be important. In fact, reinterpreting some of the existing monolepton $[173,174]$ and mono-photon [175] searches could be useful in further constraining the mono-W and mono-photon final states. This point is left for further investigation. Such channels could thus indirectly constrain scenarios with light stop masses beyond the current reaches of direct stop searches. Note that for all topologies involving virtual W's or Z's, it is the hadronic decays of the gauge bosons that dominate. However, the leptonic decays will also be present with smaller $\sigma \times \mathcal{B}$ but can in principle have higher sensitivity than the hadronic channels.

As explained at the beginning of this section, each SUSY point leads to more than one missing SMS topology, a list of up to 10 such topologies is available from SModELS. In figure 4, the topologies with the highest cross sections are described. The question of which other topologies occur in this scenario, and whether they lead to some more sensitive final states still needs to be answered. In order to illustrate this, all topologies with a $\sigma \times \mathcal{B}>1 \mathrm{fb}$ are sorted according to their frequency of occurrence and the seven most frequent topologies are shown in figures 5 and figure 6 . Once again, in order to make it easy to understand the origin and correlation of the topologies, only topologies originating from stop/sbottom decays are plotted in figure 5 in the $m_{\widetilde{b}_{1}}-m_{\widetilde{\chi}_{1}^{0}}$ plane, while those from the decays of electroweakinos are plotted in figure 6. Comparing figure 5 to the top panel of figure 4 it is clear that along with the dominant topologies, (b) ( $t$ ) and those involving hadronic $\mathrm{W}^{*} / \mathrm{Z}^{*}$ decays that are found in both figures, it is also possible to find other topologies which might have a better signal to background ratio e.g. (b) (b, $\left.Z_{\text {lep }}^{*}\right)$ or $(b)(b, \gamma)$. The latter again occurs from the loop-induced decay of the second neutralino produced in sbottom decay as explained in table 3 . This topology with $2 \mathrm{~b}$ 


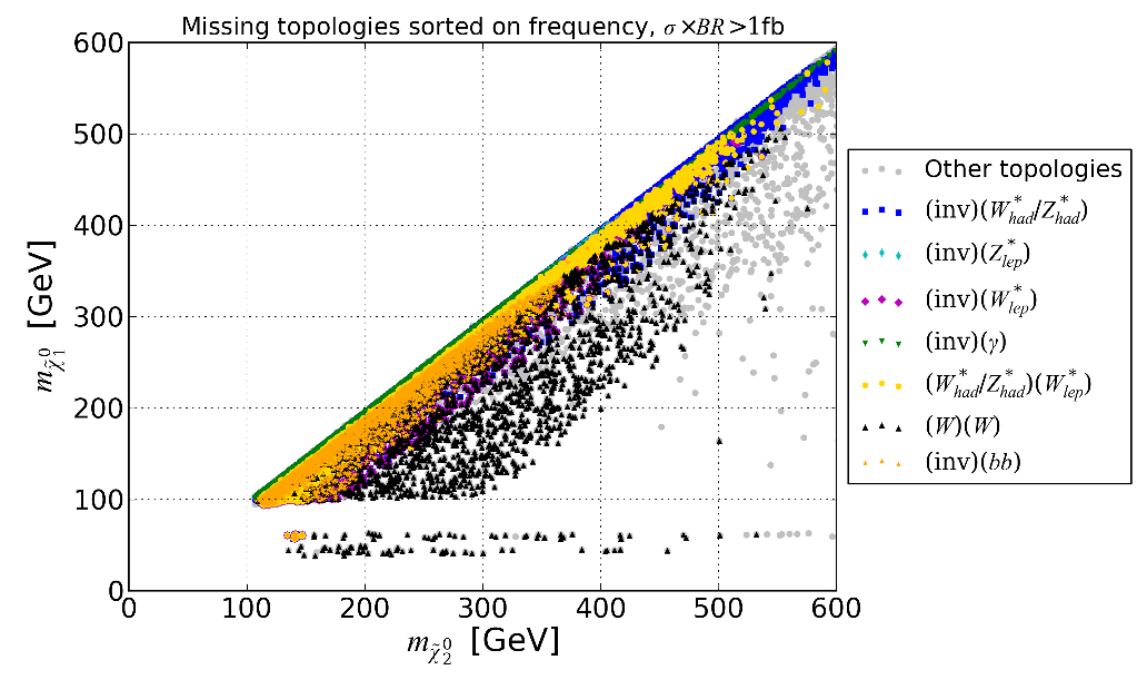

Figure 6. The figure shows missing topologies with cross section greater than $1 \mathrm{fb}$ originating due to electroweakino decays sorted on their frequency of occurrence. The minimum cross section of any topology plotted is $1 \mathrm{fb}$. The seven most frequently occurring topologies are illustrated. Topologies originating from stop/sbottom decays are suppressed.

jets, 1 photon + MET in the final state can be used to constrain the kinematic edge with $m_{\widetilde{b}_{1}}-m_{\widetilde{\chi}_{1}^{0}}<50 \mathrm{GeV}$.

Missing topologies in figure 5 show a strong correlation with the sbottom mass. Topologies involving two b jets, MET and a virtual gauge boson originating from the decay of sbottoms into a heavier neutralino/chargino occur mostly in the region with a small difference between the sbottom and the LSP. Only a few topologies involving stop pair production extend beyond $m_{\widetilde{b}_{1}}>800 \mathrm{GeV}$ where the sbottom pair production becomes too small.

In figure 5, it is difficult to highlight topologies originating from the decays of stops. The reason is the large mixing angle in the stop sector due to the Higgs mass constraints. In this case, the stop has no preferred decay channel, thus, missing topologies with a large $\sigma \times \mathcal{B}$ which naturally result from a large branching ratio in a single channel are absent.

As the $m_{\widetilde{\chi}_{1}^{ \pm}}$and $m_{\widetilde{\chi}_{1}^{0}}$ are extremely mass degenerate, we plot the missing topologies in the electroweak sector in the $\widetilde{\chi}_{2}^{0}-\widetilde{\chi}_{1}^{0}$ plane. Figure 6 shows that most of the topologies in this sector are in fact off-shell decays of $W$ and $Z$ bosons associated with MET. Clearly, in most of the region where hadronic decays of $\mathrm{W}$ or $\mathrm{Z}$ are dominant, the leptonic decays are also present. Moreover the importance of the diboson missing topology is once again evident. Expectedly, in the region with $m_{\widetilde{\chi}_{2}^{0}}, m_{\widetilde{\chi}_{1}^{0}} \gtrsim 400 \mathrm{GeV}$, the leptonic decays become irrelevant due to low $\sigma \times \mathcal{B}$.

\subsection{Stop NLSP at LHC $14 \mathrm{TeV}$}

In this section we focus on the region of parameter space where the lightest top squark is the NLSP and has a small mass difference with the LSP hence the decay $\widetilde{t}_{1} \rightarrow t \widetilde{\chi}_{1}^{0}$ is not open. If the mas gap $m_{\widetilde{t}_{1}}-m_{\widetilde{\chi}_{1}^{0}} \lesssim 85 \mathrm{GeV}$ (which is true for most of the allowed points with 

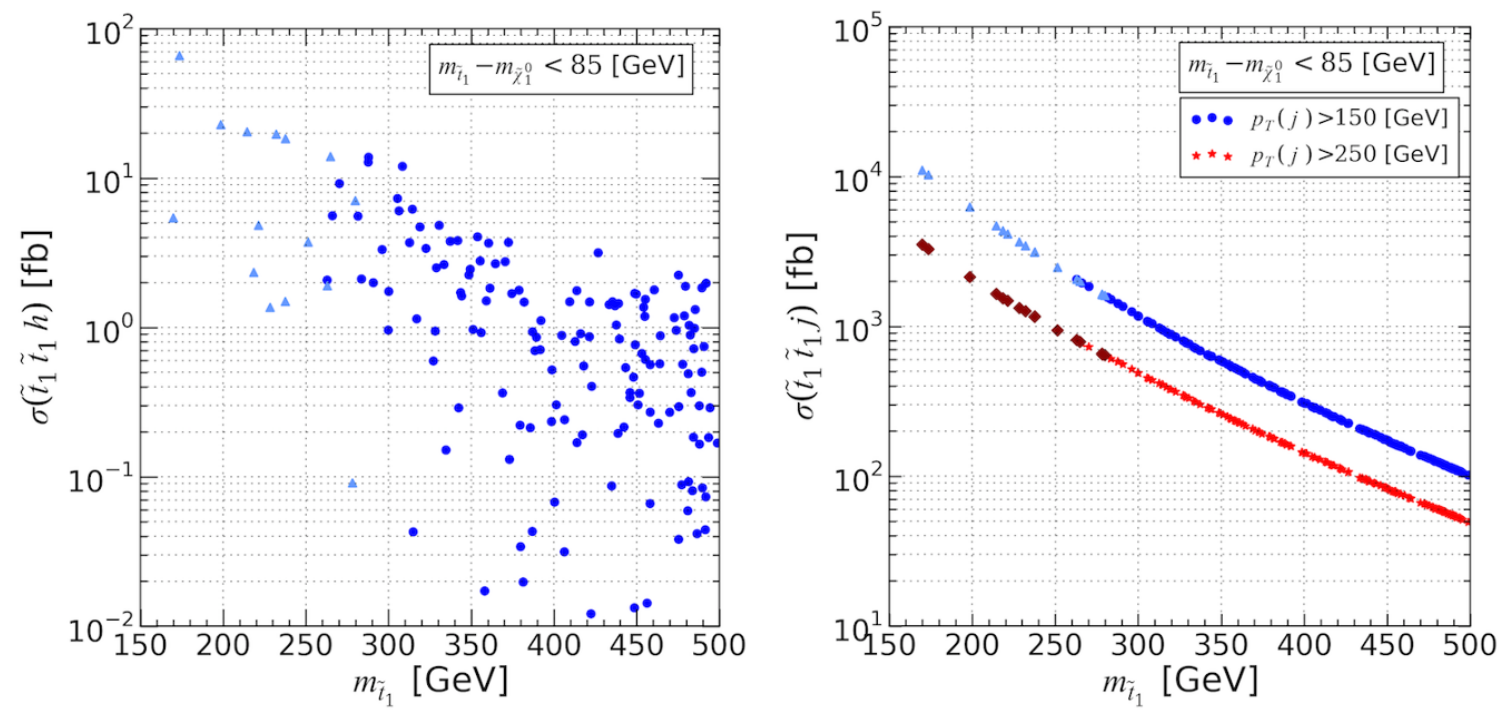

Figure 7. Leading order cross sections for $\widetilde{t}_{1} \widetilde{t}_{1} j$ and $\widetilde{t}_{1} \widetilde{t}_{1} h$ at the $14 \mathrm{TeV}$ LHC. MadGraph5_aMC@NLO version 2.2.2 [176] was used for the computation. The triangles represent points that are potentially excluded from ATLAS searches for $\widetilde{t}_{1} \rightarrow c+\widetilde{\chi}_{1}^{0}$ and stop four body decays.

a stop NLSP) then the 3-body decay $\widetilde{t}_{1} \rightarrow b W^{+} \widetilde{\chi}_{1}^{0}$ is kinematically forbidden and the only allowed decay modes are the 4-body decay $\widetilde{t}_{1} \rightarrow b \widetilde{\chi}_{1}^{0} f f^{\prime}$ and the flavour violating decay $\widetilde{t}_{1} \rightarrow c / u \widetilde{\chi}_{1}^{0}$. Because of the small mass gap between the light stop and the LSP, the decay products are very soft on average making this scenario extremely challenging for the LHC searches. This explains the very low lower bound on the light stop mass $\left(m_{\widetilde{t}_{1}} \gtrsim 275 \mathrm{GeV}\right)$ obtained with the LHC $8 \mathrm{TeV}$ data for this specific scenario. Hence, it is interesting to explore the prospect of the $14 \mathrm{TeV}$ LHC for this scenario. It was shown that using monojet + large missing transverse energy final state a stop mass $\gtrsim 300 \mathrm{GeV}$ will not be ruled out even with $100 \mathrm{fb}^{-1}$ integrated luminosity at $14 \mathrm{TeV}$ LHC [75]. The same question was also investigated in ref. [80] using $\alpha_{T}$ and $M_{T 2}$. Assuming $\mathcal{B}\left(\widetilde{t}_{1} \rightarrow c \widetilde{\chi}_{1}^{0}\right)=1$ it was shown that the exclusion limit can be extended to a maximum of $\sim 450 \mathrm{GeV}$ (depending on the mass gap between $\widetilde{t}_{1}$ and $\widetilde{\chi}_{1}^{0}$ ) with $100 \mathrm{fb}^{-1}$ data. Moreover, the use of charm-tagging could prove very useful in the future [80].

As there are not many handles to suppress the SM backgrounds in the stop NLSP scenario, the radiation of a light Higgs from one of the stops in stop pair production could provide an additional handle to discriminate the signal. It was shown in $[177,178]$ that the associated production of a stop pair with a light Higgs could be large in some region of parameter space, basically due to a large $\widetilde{t}_{1} \widetilde{t}_{1} h$ coupling. However, when taking into consideration the mass of the Higgs which requires a contribution from the stops and maximal mixing, the $\widetilde{t}_{1} \widetilde{t}_{1} h$ vertex is suppressed, hence the $\widetilde{t}_{1} \widetilde{t}_{1} h$ cross section is expected to be quite low. Moreover this process depends on the parameters of the model whereas the cross section for $\widetilde{t}_{1} \widetilde{t}_{1} j$ production is expected to be much larger and depends only on $m_{\widetilde{t}_{1}}$. In figure 7 we show $\widetilde{t}_{1} \widetilde{t}_{1} h$ (left panel) and $\widetilde{t}_{1} \widetilde{t}_{1} j$ (right panel) production cross 


\begin{tabular}{|c|c|c|c|c|c|c|}
\hline$m_{\widetilde{t}_{1}}$ & $m_{\widetilde{\chi}_{1}^{0}}$ & $m_{\widetilde{\chi}_{1}^{ \pm}}$ & $\mathcal{B}\left(\widetilde{t}_{1} \rightarrow c \widetilde{\chi}_{1}^{0}\right)$ & $\mathcal{B}\left(\widetilde{t}_{1} \rightarrow b f f^{\prime} \widetilde{\chi}_{1}^{0}\right)$ & $\mathcal{B}\left(\widetilde{t}_{1} \rightarrow b \widetilde{\chi}_{1}^{ \pm}\right)$ & $\sigma\left(\widetilde{t}_{1} \widetilde{t}_{1} h\right)(\mathrm{fb})$ \\
\hline 305.3 & 295.3 & 820.7 & 1 & 0 & 0 & 7.3 \\
\hline 372.4 & 364.0 & 1097.0 & 1 & 0 & 0 & 3.7 \\
\hline 328.8 & 301.2 & 1033.6 & 0.41 & 0.59 & 0 & 2.5 \\
\hline 314.3 & 305.1 & 309.5 & 0 & 1 & 0 & 6.2 \\
\hline 308.3 & 260.1 & 264.0 & 0 & 0 & 1 & 12.0 \\
\hline 353.7 & 319.8 & 322.8 & 0 & 0 & 1 & 4.1 \\
\hline
\end{tabular}

Table 4. A few benchmark points to show specific examples of different stop decay modes and also the $14 \mathrm{TeV} \widetilde{t}_{1} \widetilde{t}_{1} h$ production cross section in fb. While for the first three benchmarks the lightest stop is the NLSP, for the final three points the lightest chargino is the NLSP.

sections at the $14 \mathrm{TeV}$ LHC for all the allowed points with the light stop as the NLSP. The triangles represent potentially excluded points due to constraints coming from ATLAS $\widetilde{t}_{1} \rightarrow c+\widetilde{\chi}_{1}^{0}$ and stop four body decays. The production cross section for $\widetilde{t}_{1} \widetilde{t}_{1} h$ final state is at least an order of magnitude smaller than that for $\widetilde{t}_{1} \widetilde{t}_{1} j$. This makes the $\widetilde{t}_{1} \widetilde{t}_{1} h$ channel much less promising than the $\widetilde{t}_{1} \widetilde{t}_{1} j$ final state. Although there can be considerable gain in the background reduction if the Higgs in the final state is tagged but the existence of irreducible background like $p p \rightarrow h Z(\rightarrow \nu \nu)$ (with a $14 \mathrm{TeV}$ cross section $\sim 150 \mathrm{fb}$ ) makes it extremely challenging. For definiteness, in table 4 we provide a few example benchmarks with different $\widetilde{t}_{1}$ decay modes. We avoid a choice of benchmark point within the excluded regions of the existing ATLAS $\widetilde{t}_{1} \rightarrow c \widetilde{\chi}_{1}^{0}$ or stop four body decays.

In the first two benchmarks $\widetilde{t}_{1}$ exclusively decays to $c \widetilde{\chi}_{1}^{0}$ and in the following two points $\widetilde{t}_{1}$ exclusively decays to the 4-body final state. In the final two benchmarks $\widetilde{t}_{1}$ is not the NLSP - the $\widetilde{\chi}_{1}^{ \pm}$and $\widetilde{\chi}_{2}^{0}$ lie beneath $\widetilde{t}_{1}$ in the spectrum. As a consequence, the $\widetilde{t}_{1}$ exclusively decays to $b \widetilde{\chi}_{1}^{ \pm}$. The chargino in this case will eventually decay to $f f^{\prime} \widetilde{\chi}_{1}^{0}$ giving rise to again a 4-body decay of $\widetilde{t}_{1}$ but because of the existence of an on-shell chargino in the decay chain, the kinematics will be different. In summary, the stop pair production in association with an extra hard jet will be the most promising channel for probing the stop NLSP region with small stop-neutralino mass gap. The prospect of stop pair production process associated with a Higgs boson does not look encouraging mainly because of the small production cross section. We find that with the Higgs mass constraint, the stop composition is such that the $\widetilde{t}_{1} \widetilde{t}_{1} h$ coupling is small. This means that the $\widetilde{t}_{1} \widetilde{t}_{1} h$ cross-section is suppressed even for light stops, the suppression being dynamic and not so much due to kinematics. The existence of a Higgs in the final state may provide an additional handle to combat backgrounds, hence a more focused study may be worthwhile. As far as the decay of stop is concerned, there are three distinct categories where the final state objects and/or the kinematics are different. Hence, dedicated searches at the $14 \mathrm{TeV}$ LHC for each of them should be carried out.

\subsection{Decays of heavier stop}

Searches for the heavier stop could provide an alternative for probing the light stop scenarios, the main issue for exploiting these searches at the LHC remains the mass scale of the heavier stop. Due to the large radiative corrections required to achieve the correct 

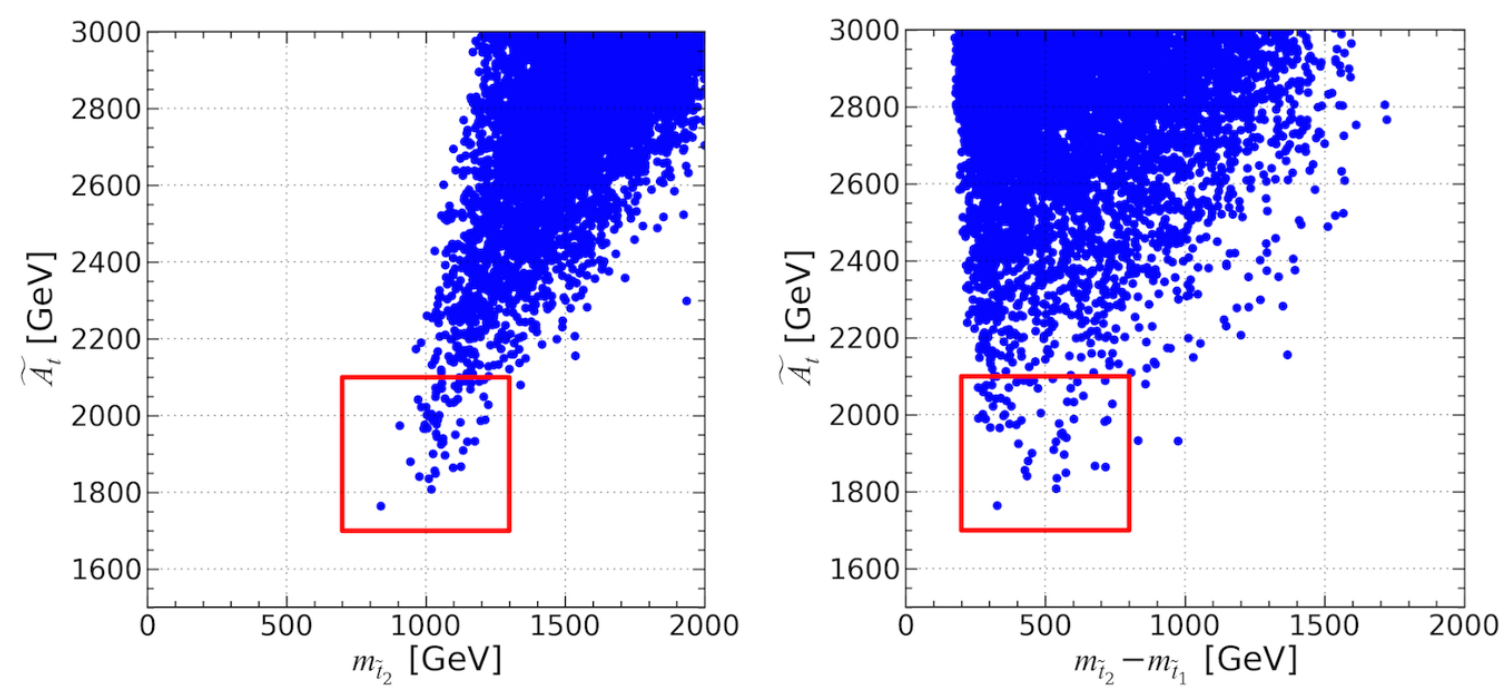

Figure 8. The allowed pMSSM points in the $\tilde{A}_{t}-m_{\widetilde{t}_{2}}$ (left panel) and $\tilde{A}_{t^{-}}\left(m_{\widetilde{t}_{2}}-m_{\widetilde{t}_{1}}\right)$ (right panel) planes.

Higgs mass in the MSSM either large $A_{t}$ or heavy stop masses is required. A large value of $A_{t}$ introduces a large splitting in stop masses and pushes the heavier stop above the $\mathrm{TeV}$ scale. In the left panel of figure 8 we plot the allowed points in the $\tilde{A}_{t}-m_{\widetilde{t}_{2}}$ plane, while in the right panel the same set of points are plotted against the mass difference between two stops $\left(m_{\widetilde{t}_{2}}-m_{\widetilde{t}_{1}}\right)$. Recall that $\tilde{A}_{t}$ is related to the naturalness of the SUSY spectrum (see eq. (1.4)). Figure 8 shows that the value of $\tilde{A}_{t}$ is constrained to be approximately above $1.8 \mathrm{TeV}$. This, in turn, gives a lower bound on the fine-tuning parameter $\Delta \gtrsim 50$ (assuming $\Lambda_{\text {mess }}=20 \mathrm{TeV}$ ) which amounts to $\sim 2 \%$ tuning.

An optimistic scenario with $\Delta \sim 50$ leads to $m_{\widetilde{t}_{2}} \sim 1 \mathrm{TeV}$ and $\left(m_{\widetilde{t}_{2}}-m_{\widetilde{t}_{1}}\right) \sim 500 \mathrm{GeV}$ (the region enclosed by the thick red line). Thus, an interesting outcome of our analysis is the possibility of a SUSY spectrum with a heavier stop mass around a $\mathrm{TeV}$ with a large mass gap $(\sim 500 \mathrm{GeV})$ with the lighter stop. The discovery potential of this scenario at the $14 \mathrm{TeV}$ LHC will in general depend on the decay channel of $\widetilde{t}_{2}$ which in turn depends on the masses of the other SUSY particles. We will consider two specific decay modes $\widetilde{t}_{2} \rightarrow \widetilde{t}_{1} Z$ and $\widetilde{t}_{2} \rightarrow \widetilde{t}_{1} h$ which are particularly interesting and have in general large branching ratios (as we will show below). The couplings $\widetilde{t}_{2}-\widetilde{t}_{1}-Z$ (in the limit when the mass splitting between $\widetilde{t}_{2}$ and $\widetilde{t}_{1}$ is large and $\left.M_{A}, M_{H} \gg M_{Z}\right)$ and $\widetilde{t}_{2}-\widetilde{t}_{1}-h$ can be written as $[177,178]$,

$$
\begin{aligned}
& \lambda_{\widetilde{t}_{2} \widetilde{t}_{1} Z} \approx \frac{g}{2 M_{W}} m_{t} X_{t}, \\
& \lambda_{\widetilde{t}_{2} \widetilde{t}_{1} h} \approx 2\left(\sqrt{2} G_{F}\right)^{\frac{1}{2}} M_{Z}^{2}\left[\left(\frac{2}{3} \sin ^{2} \theta_{W}-\frac{1}{4}\right) \cos (2 \beta) \sin \left(2 \theta_{t}\right)+\frac{1}{2} \frac{m_{t}}{M_{Z}^{2}} \cos \left(2 \theta_{t}\right) X_{t}\right]
\end{aligned}
$$



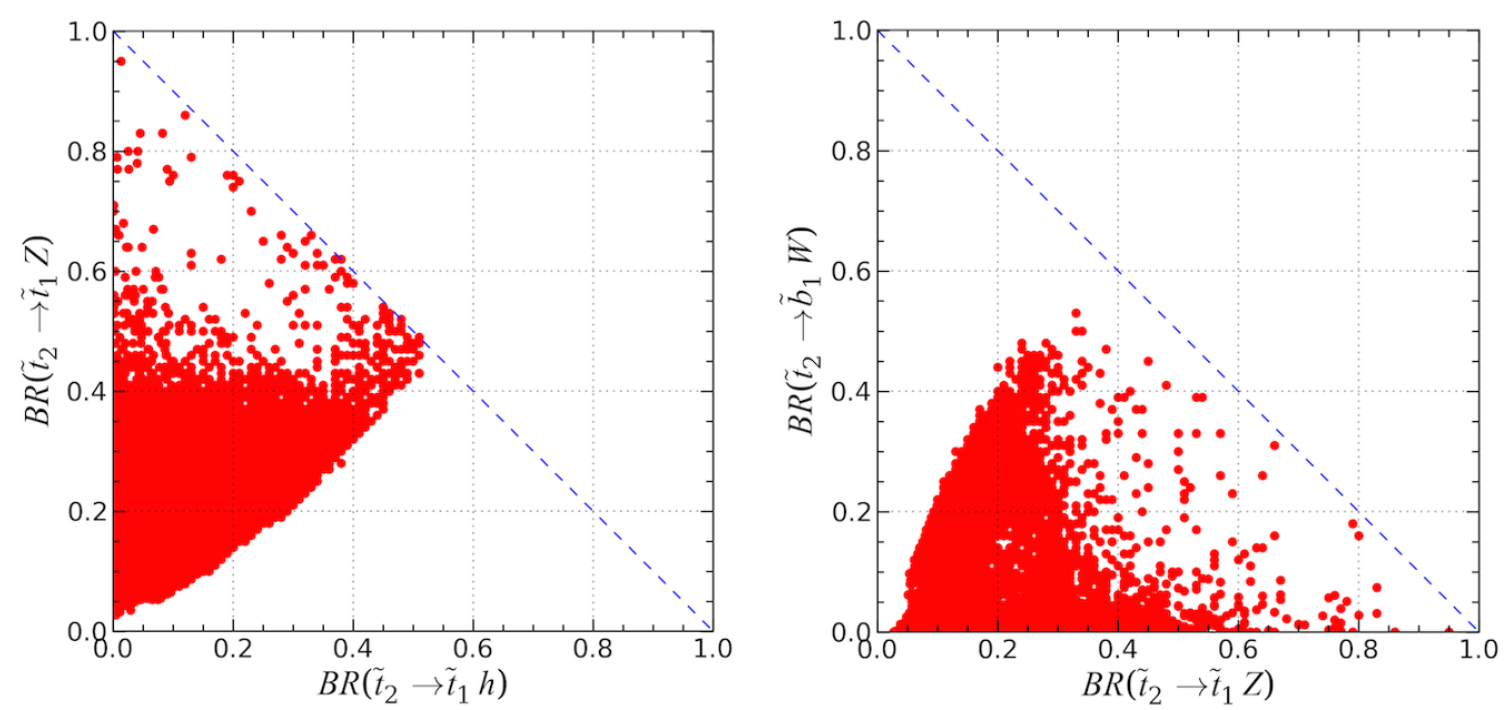

Figure 9. Left panel: branching ratio of $\widetilde{t}_{2} \rightarrow \widetilde{t}_{1} h$ vs. that of $\widetilde{t}_{2} \rightarrow \widetilde{t}_{1} Z$. Right panel: branching ratio of $\widetilde{t}_{2} \rightarrow \widetilde{t}_{1} Z$ vs. that of $\widetilde{t}_{2} \rightarrow \widetilde{t}_{1} W$. The region only below the blue dashed line is physical.

The mixing angle between the left and right handed top squarks, $\theta_{t}$ and the mixing parameter $X_{t}$ are defined through the mass matrices [177],

$$
\begin{aligned}
M_{\widetilde{t}}^{2} & =\left(\begin{array}{cc}
m_{L L}^{2}+m_{t}^{2} & m_{t} X_{t} \\
m_{t} X_{t} & m_{R R}^{2}+m_{t}^{2}
\end{array}\right) \\
X_{t} & =A_{t}-\frac{\mu}{\tan \beta} \\
m_{L L}^{2} & =m_{\widetilde{Q}_{3}}^{2}+\left(\frac{1}{2}-\frac{2}{3} \sin ^{2} \theta_{W}\right) \cos 2 \beta M_{Z}^{2} \\
m_{R R}^{2} & =m_{\widetilde{U}_{3}}^{2}+\frac{2}{3} \sin ^{2} \theta_{W} \cos 2 \beta M_{Z}^{2} \\
m_{\tilde{t}_{1,2}}^{2} & =m_{t}^{2}+\frac{1}{2}\left[m_{L L}^{2}+m_{R R}^{2} \mp \sqrt{\left(m_{L L}^{2}-m_{R R}^{2}\right)^{2}+4 m_{t}^{2} X_{t}^{2}}\right] \\
\sin 2 \theta_{t} & =\frac{2 m_{t} X_{t}}{m_{\widetilde{t}_{1}}^{2}-m_{\widetilde{t}_{2}}^{2}}, \quad \cos 2 \theta_{t}=\frac{m_{L L}^{2}-m_{R R}^{2}}{m_{\widetilde{t}_{1}}^{2}-m_{\widetilde{t}_{2}}^{2}} .
\end{aligned}
$$

Eq. (6.1) shows that the $\widetilde{t}_{2}-\widetilde{t}_{1}-Z$ coupling will be large if $X_{t}$ is large (which is required for the Higgs mass unless both stops are extremely heavy). The $m_{t}$ enhancement can also be understood by noting that in the large energy limit (large mass splitting between $\widetilde{t}_{2}$ and $\tilde{t}_{1}$ ) the $Z$ boson can be replaced by one of the would-be goldstone boson using the goldstone boson equivalence theorem. The $\widetilde{t}_{2}-\widetilde{t}_{1}-h$ coupling has slightly more structure and has both the F-term and D-term contributions. However, this coupling can also be quite large in some part of the parameter space. Hence, these two decay modes are well motivated from the fact that large $X_{t}$ is required for the Higgs mass. Of course, the presence of other particles below the $\widetilde{t}_{2}$ mass makes the picture more complicated and many other decay modes can contribute. In figure 9 we show the branching ratios into gauge and Higgs 
boson for all the allowed points. One can see that for a large fraction of points the sum of $\mathcal{B}\left(\widetilde{t}_{2} \rightarrow \widetilde{t}_{1} Z\right)$ and $\mathcal{B}\left(\widetilde{t}_{2} \rightarrow \widetilde{t}_{1} h\right)$ is quite large supporting our analytic expectation.

Hence, the pair production of $\widetilde{t}_{2}$ with the subsequent decays $\widetilde{t}_{2} \rightarrow \widetilde{t}_{1} Z$ and $\widetilde{t}_{2} \rightarrow \widetilde{t}_{1} h$ can be a very interesting channel to look at. The $14 \mathrm{TeV}$ prospect of this scenario was studied in [79] by one of the authors. It was shown that a $4-5 \sigma$ signal can be observed for $\sim 1 \mathrm{TeV} \widetilde{t}_{2}$ with $100 \mathrm{fb}^{-1}$ integrated luminosity. We refer the readers to [79] for further details.

\subsection{Decays of heavy Higgs}

In this section we turn our attention to the neutral heavy Higgs bosons $H$ and $A$. An important result of our analysis is that the mass of the heavy neutral scalar $H$ is constrained to be larger than $\sim 450 \mathrm{GeV}$. Note that this lower bound arises only after imposing constraints from the light Higgs signal strengths and Heavy Higgs searches implemented in HiggsSignals and HiggsBounds. Moreover the $A$ is quite degenerate with the $H$, the mass difference being almost always less than $5 \mathrm{GeV}$, which is less than one percent of the common mass. For such heavy Higgses, the widths are $\sim 0.05 m_{H}, m_{A}$ from SM fermions alone [179]. These widths can only increase when decay channels into sparticles are included. Thus the mass difference between $H$ and $A$, is always comparable or smaller than their widths. It is well known that the agreement of the observed signal strengths with those expected in the SM, actually forces the global fits to the alignment region where $|(\beta-\alpha)| \sim \frac{\pi}{2}$. As a result the gauge boson couplings are severely suppressed for the $H$. Hence, even with the large mass of $H$ and the enhancement factor in the $V V$ decay width due to the decays in the longitudinal $V$ bosons, the branching ratio into vector bosons is not above one percent. Recall that the $A V V$ vertex is absent at tree level. We have also checked that the decays $H \rightarrow h h$ and $A \rightarrow Z h$ have branching ratios smaller than one percent for all our points. Thus, the only relevant tree level decay modes can be into standard model fermions and sparticles: the sfermions and the electroweakinos. Moreover the branching ratios should be similar for the $H$ and the $A$.

The large mass of the $H$ means that the decay to the $t \bar{t}$ final state is now kinematically allowed. As a result it can be the dominant decay mode for small values of $\tan \beta$ and the $\mathcal{B}(H \rightarrow t \bar{t})$ can be as high as $\geq 80 \%$ for $\tan \beta \lesssim 5$. With increasing values of $\tan \beta, \mathcal{B}(H \rightarrow$ $t \bar{t})$ drops gradually and the $H \rightarrow b \bar{b}$ decay mode starts dominating. These two branching ratios sum up to about $80 \%$ in large part of the parameter space. The remaining $20 \%$ is mostly taken up by $\mathcal{B}(H \rightarrow \tau \bar{\tau})$ decays. The SM decay modes are however suppressed when decay channels into SUSY particles become important as will be discussed below. In the left panel of figure 10 we show the $14 \mathrm{TeV}$ cross sections for $H$ production $\left(\sigma_{14 \mathrm{TeV}}^{H}\right)$ times the branching ratio to the $\tau \tau$ and $t \bar{t}$ final states for the allowed points for which $\sigma_{14 \mathrm{TeV}}^{H} \times \mathcal{B}(H \rightarrow f \bar{f})$ is greater than $1 \mathrm{fb}$. The production cross section were computed with SusHi-1.5.0 [180-188]. The decays of $H / A$ into $\tau$ pair clearly offer a search channel with $\sigma_{14 \mathrm{TeV}}^{H} \times \mathcal{B}$ that can reach $\mathcal{O}(10) \mathrm{fb}$, for $\tan \beta \lesssim 20$ and $m_{H} \sim 1 \mathrm{TeV}$. For the $t \bar{t}$ final state $\sigma_{14 \mathrm{TeV}}^{H} \times \mathcal{B}(H \rightarrow t \bar{t})$ can be as high as 10's of fb and higher for low $\tan \beta \lesssim 15$ and $m_{H} \lesssim 1000 \mathrm{GeV}$. 

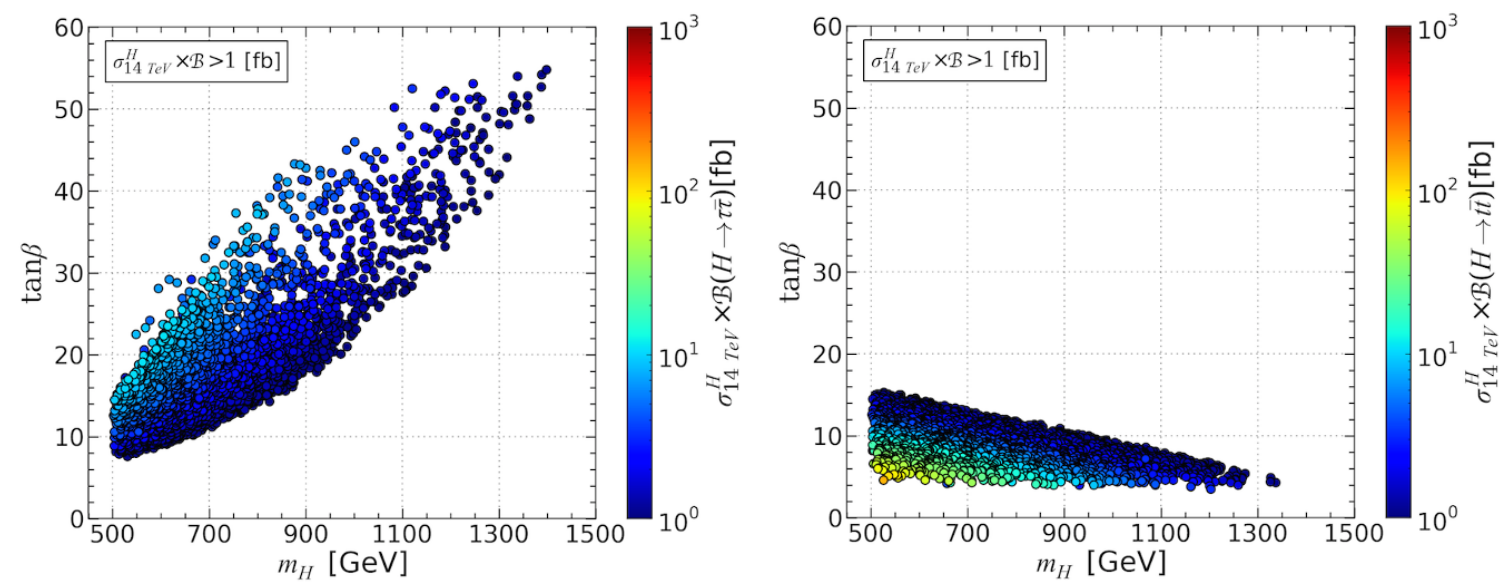

Figure 10. The product of $14 \mathrm{TeV}$ cross section of the CP-even heavy Higgs and its branching ratio to $\tau \tau$ final state (left panel), t $t \bar{t}$ final state (right panel) in the $\tan \beta-M_{A}$ plane.
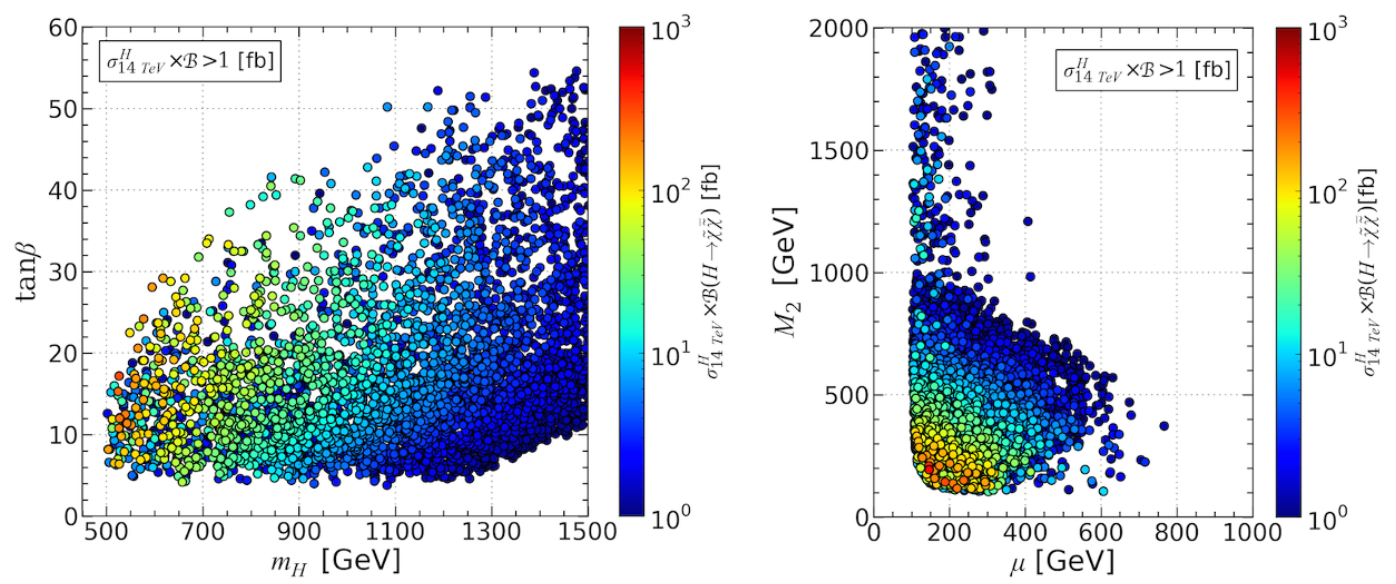

Figure 11. The product of $14 \mathrm{TeV}$ cross section of the CP-even heavy Higgs and its branching ratio to electroweakino final state in the $m_{H}$-tan $\beta$ plane (left panel) and in the $M_{2}-\mu$ plane (right panel).

The decay of the heavy Higgs $H$ to SUSY final states can also be quite important. In figure 11 we plot the product of cross section times branching ratio for $H$ to the electroweakino final states. ${ }^{6}$ All channels where the heavy Higgs can decay to charginos or neutralinos are summed over. The 'invisible' decays of the $H$ include $\widetilde{\chi}_{1}^{0} \widetilde{\chi}_{1}^{0}$ as well as the decay into heavier states when the mass difference between the sparticle (eg. $\left.\widetilde{\chi}_{2}^{0}\right)$ and the LSP is small: $\lesssim 5 \mathrm{GeV}$. We find that the total 'invisible' branching ratio of the $H$ is always less than $30 \%$ and mostly less than $10 \%$ while the visible decays into electroweakinos can reach $80 \%$. The branching ratios for the $A$ are also similar to those for the $H$ presented in the figure. In fact, apart from the production cross section, the phenomenology of $H$ and $A$ for these allowed points are rather similar. It is also well known that for the same mass,

\footnotetext{
${ }^{6}$ There is a small fraction of $H$ that decays to light stops as well. However, this channel is not always kinematically open and most of the times $\mathcal{B}\left(H \rightarrow \widetilde{t}_{1} \widetilde{t}_{1}^{*}\right)<0.1$.
} 
the gluon fusion production cross section can be higher for $\mathrm{A}$ than for $\mathrm{H}$, the exact values depending on the masses of the squarks.

There have been dedicated discussions of the Higgs sector of the MSSM, allowed after the LHC Run-I, beginning from the analysis of the partial first data set [189] in the pMSSM framework to the more recent analyses $[179,190]$ in the hMSSM and pMSSM scenarios respectively which focus on searches for the Heavy Higgses at the LHC $14 \mathrm{TeV}$.

Obviously, as the above discussion shows the $t \bar{t}$ final state is perhaps the most crucial in the low $\tan \beta$ range and the $\tau \bar{\tau}$ in the large $\tan \beta$ range. While the $\tau \bar{\tau}$ channel has received a lot of attention in the past, the LHC Run-I results [191] have forced attention to be focused on the $t \bar{t}$ final state. It has been known since a long time that for this final state, interference with the $t \bar{t}$ QCD background gives a very characteristic peak-dip structure [192, 193]. The feasibility of using it to isolate the signal from the background as well as the difference in the spin spin correlations between the $t$ and $\bar{t}$ for the background and the resonant signal have been discussed in the literature [194-196]. In the CP conserving case, the $H$ and $A$ amplitudes do not interfere but still the presence of almost degenerate $H, A$ can degrade the effect. The peak-dip structure seems to be subdominant to the effect of higher order corrections [197] and more intricate cuts may have to be devised to enhance the resonant Higgs contribution. Moreover a recent analysis [198] concluded that this peak-dip structure will get degraded due to the limited resolution of the $t \bar{t}$ invariant mass, the statement being even more true with the presence of degenerate $H$ and $A$ as would be the case here. Hence kinematic cuts which exploit the effect of the spin-spin correlations for the $t \bar{t}$ produced in the $H$ decay would be necessary (see for example [199]). A simple analysis of [179] which includes such cuts, shows that at the LHC $14 \mathrm{TeV}$ one could be sensitive to $\tan \beta \sim 6$ for $m_{H} \sim 500 \mathrm{GeV}$ and to $\tan \beta \sim 1$ for $m_{H} \sim 1 \mathrm{TeV}$. More analyses, to improve the sensitivity of this channel are required, see for example [198, 200].

Of some interest are the decays into the electroweakinos where the product of cross section times branching ratios can reach values as high as $10^{2}$ to $10^{3} \mathrm{fb}$ for $H$ masses up to $700-1000 \mathrm{GeV}$ and $\tan \beta \lesssim 20-30$. Thus we find that this channel can offer interesting search possibility for the $H / A$. Note that the general conclusion of [179] that the parameter ranges which gives large branching into these channels have been ruled out by the LHC trilepton constraints does not apply in our analysis where $M_{1}, M_{2}$ values are not related. The large values of the cross section times branching are concentrated in the low $M_{2}-\mu$ region and hence the chargino/neutralinos produced are likely to give rise to final states with real or virtual $W / Z$. However, the topology for final states resulting from the $H$ decay into electroweakinos, followed by their cascade decays, will depend on their couplings and on the masses which are strongly influenced by the relic density upper limit. A detailed investigation of the potential to use electroweakino decay channels to extend the reach for heavy Higgs, a topic that was hardly addressed [201] is beyond the scope of this paper.

As mentioned already the $H$ has an invisible branching ratio which can be upto 0.3 but is mostly less than 0.1. One can in fact search 'directly' for such an invisible $H$ at the LHC via the associated production of the $H$ with a vector boson [202] or production of $H$ via the vector boson fusion [203]. Indeed currently bounds exist on the invisible branching ratio of the $126 \mathrm{GeV}$ Higgs by both the CMS [204] and ATLAS [205, 206], using 

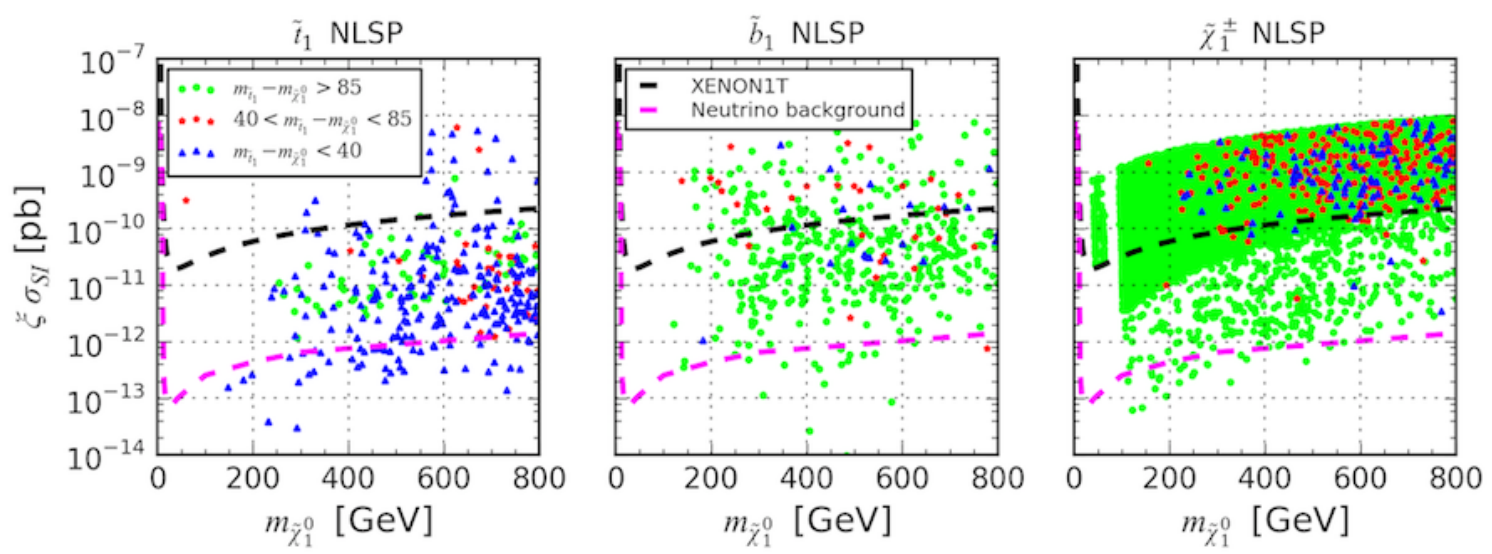

Figure 12. Spin-Independent $\widetilde{\chi}_{1}^{0}$-nucleon cross section vs. the $\widetilde{\chi}_{1}^{0}$ mass for $\widetilde{t}_{1}$ (left), $\widetilde{b}_{1}$ (center)and $\widetilde{\chi}_{1}^{ \pm}$(right) NLSP. For comparison, the expected limits from XENON1T and the neutrino coherent scattering are superimposed.

both the modes. For a $120 \mathrm{GeV}$ Higgs boson, the $14 \mathrm{TeV}$ LHC should be able to probe a value of the branching ratio as low as 0.17 [207]. Earlier projections [202, 203] had looked at larger values of the scalar mass. Unfortunately, these channels will be of not much use in the present case for the $H$ as its couplings to a $V V$ pair are highly suppressed. Hence the production of $H$ in gluon fusion with associated jets followed by the decay of $H$ in invisible channel [208] or associated production of $H$ with a $t \bar{t}$ pair followed by an invisible decay of the $H$, are the two possibilities for such an invisibly decaying $H$. The estimates of the expected rates for the latter channel presented in [198] assuming an invisible branching ratio of 1 , shows that the search in the channel $t \bar{t}$ pair + MET will be a challenging one. As far as an invisibly decaying $A$ is concerned, for the same mass and coupling strengths, the associated production will have smaller rates for $t \bar{t} A$ compared to $t \bar{t} H$ (see for example [209, 210]). Hence production of the (pseudo) scalar in gluon fusion with associated jets will be a better channel to probe for such an invisibly decaying $A$. Clearly, more studies are required.

\section{Complementarity with dark matter searches}

The spin-independent (SI) neutralino-proton cross section is displayed in figure 12 for the cases of the $\widetilde{t}_{1}, \widetilde{b}_{1}$ and $\widetilde{\chi}_{1}^{ \pm}$NLSPs. The points are color coded according to the stop-LSP mass difference. Although this quantity is not directly relevant for direct detection it is useful to highlight the complementarity with collider searches. Here the cross section is rescaled according to eq. (3.7) to take into account scenarios where the neutralino is only a part of the DM.

The results can be understood knowing that the predictions for the SI cross section are basically governed by the nature of the neutralino, pure states leading to small cross sections and mixed Higgsino/gaugino to the largest. A large fraction of the points will be probed by Xenon1T, in particular those with a chargino NLSP — which constitute the largest sample - since they are typically associated with a dominantly, yet mixed, 

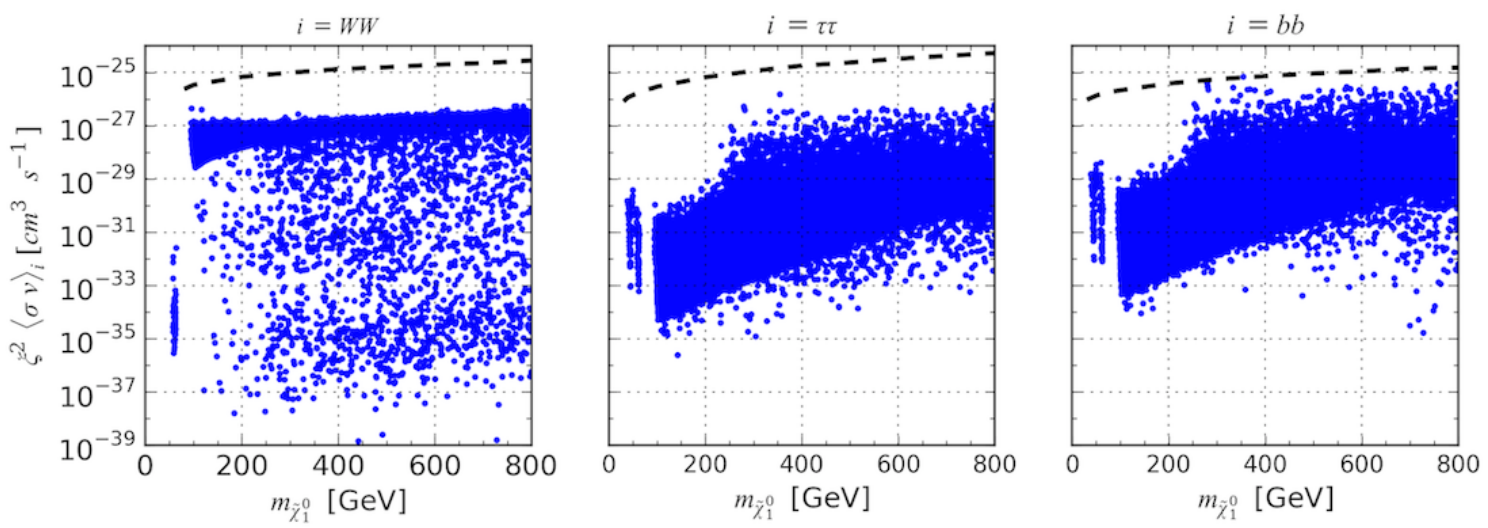

Figure 13. Indirect detection cross section as a function of LSP mass in the $W W, \tau \tau$ and $b b$ final states. For comparison the current FermiLAT [120] limit is superimposed.

Higgsino(wino) LSP. The right panel of figure 12 shows that Xenon1T has the potential to cover the vast majority of points where $40 \mathrm{GeV}<m_{\widetilde{t}_{1}}-m_{\widetilde{\chi}_{1}^{0}}<85 \mathrm{GeV}$. Those are the points that are not well constrained by current LHC bounds because the stop decays mostly into $b \widetilde{\chi}_{1}^{ \pm}$and the chargino in turn decays via a virtual W. Some of the points with $m_{\widetilde{t}_{1}}-m_{\widetilde{\chi}_{1}^{0}}<40 \mathrm{GeV}$ can also be probed by Xenon1T provided the chargino is the NLSP. Furthermore the scenarios with a LSP with a mass around 45 or $60 \mathrm{GeV}$ (corresponding to the so-called bino branches mentioned in previous sections) should be entirely probed with Xenon-1T. A few points with chargino NLSP lie below the coherent neutrino scattering background, these are typically associated with a pure Higgsino or wino LSP.

Less promising for direct detection are scenarios with a squark NLSP. Figure 12, left and center panels, shows that only a small fraction of the points will be probed by the future Xenon1T detector, a few points even lying below the irreducible coherent neutrino scattering background. In particular some of the points where $m_{\widetilde{t}_{1}}-m_{\widetilde{\chi}_{1}^{0}}<40 \mathrm{GeV}$, in the left panel of figure 12, can lead to a very small cross section. The reason is that for such mass splitting the value of the relic density is governed by the coannihilation channels with stops, hence a dominantly bino LSP is allowed. Its weak coupling to the Higgs lead to a suppressed SI cross section. Thus these points that are hard to probe at the LHC can also evade direct DM searches. Note that going beyond the standard cosmological scenario by assuming that DM can be regenerated, for example from decay of moduli fields re-injecting neutralinos after the freeze-out [211-213], such that $\xi=1$ would lead to much stronger constraint [214]. Many of the scenarios would already be constrained by LUX and nearly all of the ones with chargino NLSP could be probed at Xenon1T.

We have also computed the indirect detection cross section for LSP annihilation into $\tau^{+} \tau^{-}, b \bar{b}$ and $W^{+} W^{-}$and compared this with the exclusion obtained by FermiLAT from observations of the photon flux from dwarf spheroidal galaxies (dSphs) of the Milky Way [120]. Figure 13 shows the results for different channels after rescaling by $\xi^{2}$. Since a large fraction of the points have $\xi<1$, the rescaled cross section is often strongly suppressed, hence only a handful of points in the $b \bar{b}$ channel are excluded by such searches 
- basically those where the cross section is enhanced by annihilation through a heavy Higgs. Again assuming $\xi=1$ would lead to a completely different picture, with a strong increase in the predictions of the cross sections. In particular $\sigma v$ for DM annihilation in the WW channel exceeds the FermiLAT limit for most of the scenarios with a LSP mass below $300 \mathrm{GeV}$, in agreement with the results in $[214,215]$. It was also shown that PAMELA limits from antiprotons can constrain such scenarios [216] and that the wino can be also effectively probed by FermiLAT searches for gamma-ray lines from neutralino annihilation into photon pairs (or $\gamma Z$ ) [217].

\section{Conclusions}

The discovery of a SM-like Higgs boson has strong implications for SUSY since a Higgs mass of $126 \mathrm{GeV}$ requires large radiative corrections from the stop sector. This requires heavy stops and/or large mixing which is in conflict with the naturalness arguments. Stops therefore play a central role in SUSY and are a key ingredient in testing the naturalness of the MSSM. The LHC has performed many dedicated searches for stops. However, their production cross sections are small compared to the first two generation squarks, which leads to degraded LHC run1 limits on their masses. Hence, it is interesting to investigate to which extent light stops are still allowed and demonstrate various possible future probes of the resultant MSSM scenario at the LHC via not just the searches for the stops but also for other sparticles such as sbottoms, electroweakinos and even the heavy Higgs.

We first determined the regions of the pMSSM with ten free parameters compatible with a light stop (specifically with a mass below $1.5 \mathrm{TeV}$ ) after taking into account current constraints from the Higgs mass, Higgs signal strengths, flavour physics, the upper limit on the neutralino relic density as well as DM direct detection. Flavour constraints are very restrictive since they are in tension with the Higgs mass. For example, constraints from $B_{d} \rightarrow X_{s} \gamma$, can become stricter with large $A_{t}$, which is what the Higgs mass requires. The PLANCK upper limit on the relic density and the direct search limits from LUX are also in tension, combined they favour either TeV scale LSP or a almost pure Higgsino or wino LSP. The latter implies a supersymmetric spectra with a chargino NLSP and small mass differences between the chargino and the neutralino LSP. Another possibility is a squark NLSP (in particular a stop) since coannihilation can be used to obtain the measured value for the relic density. Both cases entail that LHC searches for SUSY are difficult.

We have then used SMODELS for all the MSSM points allowed by all the previously mentioned constraints to find implications of the limits obtained by the LHC collaborations on supersymmetric particles with the simplified model framework. We have found that stops below $500 \mathrm{GeV}$ can be consistent with all the LHC searches (including direct stop and sbottom production). The reason is either a compressed spectra or reduced branching ratios into the channels excluded with simplified models. This is our first main result.

Our results generally agree with those of [45] which also thoroughly investigates the impact of LHC SUSY searches on the pMSSM parameter space and found that light stops were allowed. Our approach however differs in two ways. First in our choice of the set of free parameters. Most importantly, in contrast to us [45] allows light sleptons and gluinos. 
Second, we impose only an upper bound on the DM relic density leading to a large fraction of points with large higgsino or wino components.

Another important aspect of our analysis is the identification of final states which would, in principle, be capable of probing the points surviving after the application of LHC, flavor, dark matter and Higgs constraints. This analysis can help determine how best to extend the search reach at the LHC, particularly for the low masses still allowed after LHC constraints. One of the most important missing topology corresponds to asymmetric stop decays - for example one of the pair produced stop decaying to $t \widetilde{\chi}_{1}^{0}$ and the other into $b \widetilde{\chi}_{1}^{ \pm}$with the chargino being invisible because it decays into the LSP and soft jets. Other possible signatures which might improve the reach involve the decay of squarks into a heavier neutralino or chargino which then decay to a LSP via virtual W/Z.

An extension of the reach in the region of small mass difference of the stop with the LSP entails using the jet and MET signature from a stop decaying into charm neutralino or via 4-body decays as done by the LHC collaborations. Although we have not used these channels as they played a minor role in our analysis, they should lead to strong constraints in the future. We also investigated whether the region near the kinematic boundary could be probed by considering associated production of stops with a Higgs. In principle tagging the Higgs allows to handle the background very well. We find that with the Higgs mass constraint, the stop composition is such that the $\widetilde{t}_{1} \widetilde{t}_{1} h$ coupling is small. As a result this cross section is small, even for small masses of the stops being considered, the suppression being dynamic and not so much due to kinematics. Hence the stop search in this channel is challenging and needs more detailed studies. The associated stop stop jet process is perhaps a better option, for the region near the kinematic boundary, though potentially more complicated to analyze. Thus the subject of associated production of a stop pair with a jet or Higgs requires further detailed studies.

Alternative probes of light stop scenarios involve search for the heavier stop through its $\widetilde{t}_{2} \rightarrow \widetilde{t}_{1} h / Z$ decay. Indeed the branching ratios for these channels in particular, for the decay $\widetilde{t}_{2} \rightarrow \widetilde{t}_{1} Z$, are expected to be large because of the observed large Higgs mass.

Interestingly, for the allowed points in our light stop scenario the heavy Higgs phenomenology is found to be very interesting. Searches of the heavy Higgses provide additional channels to probe the model, either though conventional signatures in the $\tau \tau$ decay channel or taking advantage of decays into electroweakinos, including invisible decays. Our MSSM scenarios, with no relationships between different gaugino masses, in fact allows for considerable values for these branching ratios and yet satisfy the LHC8 constraint on electroweakinos.

Finally we highlight the complementarity with DM searches, nearly all points with the stop-NLSP mass difference below the $\mathrm{W}$ mass will be tested at Xenon1T provided the NLSP is a chargino, this means that one region that is hard to cover at the LHC via squark and electroweakino searches will nicely be probed by ton scale detectors. Unfortunately, it is much harder to cover the region with a stop NLSP with direct DM searches.

In this analysis we have explicitly rejected the long-lived particles, however in our initial sample a large fraction of the points involved long-lived charged particles, in particular charginos. Existing searches for long-lived particles constrain severely the dominantly wino 
charginos in certain mass ranges [218]. Clearly improved analyses could provide a handle to probe this region of the parameter space left unexplored in this work.

Thus, we have shown that the light stops, being actively hunted at the LHC, can be probed by more than one means. While the LHC Run2 will bring interesting results and hopefully a BSM signal, possible ways of constraining the light stop scenario via indirect constraints should also be considered.

\section{Acknowledgments}

DG acknowledges support from the European Research Council under the European Union's Seventh Framework Programme (FP/2007-2013) / ERC Grant Agreement no. 279972. DG, RMG and GB wish to thank the organizers of the Workshop on High Energy Physics Phenomenology, WHEPP at Puri, India. DG would also like to thank the hospitality of LAPTh where part of this work was done. RMG wishes to acknowledge support from the Department of Science and Technology, India under Grant No. SR/S2/JCB-64/2007 under the J.C. Bose Fellowship scheme as well as support from the French ANR Project "DMAstro-LHC", ANR-12-BS05-0006, for a visit to LAPTh. SuK is supported by the "New Frontiers" program of the Austrian Academy of Sciences. We thank Jonathan Da Silva for providing us with the neutrino coherent scattering and Fermi limits. We thank Sabine Kraml, Andre Lessa, Ursula Laa, Veronika Magerl, Wolfgang Magerl, Michael Traub and Wolfgang Waltenberger for useful discussions.

Open Access. This article is distributed under the terms of the Creative Commons Attribution License (CC-BY 4.0), which permits any use, distribution and reproduction in any medium, provided the original author(s) and source are credited.

\section{References}

[1] CMS collaboration, Observation of a new boson at a mass of $125 \mathrm{GeV}$ with the CMS experiment at the LHC, Phys. Lett. B $\mathbf{7 1 6}$ (2012) 30 [arXiv:1207.7235] [INSPIRE].

[2] ATLAS collaboration, Observation of a new particle in the search for the standard model Higgs boson with the ATLAS detector at the LHC, Phys. Lett. B 716 (2012) 1 [arXiv: 1207.7214] [INSPIRE].

[3] CMS collaboration, Observation of a new boson with mass near $125 \mathrm{GeV}$ in pp collisions at $\sqrt{s}=7$ and $8 \mathrm{TeV}$, JHEP 06 (2013) 081 [arXiv:1303.4571] [INSPIRE].

[4] ATLAS collaboration, Evidence for the spin-0 nature of the Higgs boson using ATLAS data, Phys. Lett. B 726 (2013) 120 [arXiv:1307.1432] [INSPIRE].

[5] ATLAS and CMS collaborations, Combined measurement of the Higgs boson mass in pp collisions at $\sqrt{s}=7$ and $8 \mathrm{TeV}$ with the ATLAS and CMS experiments, Phys. Rev. Lett. 114 (2015) 191803 [arXiv: 1503. 07589] [INSPIRE].

[6] CMS collaboration, Precise determination of the mass of the Higgs boson and tests of compatibility of its couplings with the standard model predictions using proton collisions at 7 and $8 \mathrm{TeV}$, Eur. Phys. J. C 75 (2015) 212 [arXiv:1412.8662] [INSPIRE]. 
[7] CMS collaboration, Measurement of Higgs boson production and properties in the $W W$ decay channel with leptonic final states, JHEP 01 (2014) 096 [arXiv:1312.1129] [INSPIRE].

[8] CMS collaboration, Search for the standard model Higgs boson produced in association with a $W$ or a $Z$ boson and decaying to bottom quarks, Phys. Rev. D 89 (2014) 012003 [arXiv: 1310.3687] [INSPIRE].

[9] ATLAS collaboration, Search for $H \rightarrow \gamma \gamma$ produced in association with top quarks and constraints on the Yukawa coupling between the top quark and the Higgs boson using data taken at $7 \mathrm{TeV}$ and $8 \mathrm{TeV}$ with the ATLAS detector, Phys. Lett. B 740 (2015) 222 [arXiv:1409.3122] [INSPIRE].

[10] ATLAS collaboration, Measurements of Higgs boson production and couplings in the four-lepton channel in pp collisions at center-of-mass energies of 7 and $8 \mathrm{TeV}$ with the ATLAS detector, Phys. Rev. D 91 (2015) 012006 [arXiv:1408.5191] [INSPIRE].

[11] ATLAS collaboration, Search for the standard model Higgs boson produced in association with top quarks and decaying into b $\bar{b}$ in pp collisions at $\sqrt{s}=8 \mathrm{TeV}$ with the ATLAS detector, Eur. Phys. J. C 75 (2015) 349 [arXiv:1503.05066] [INSPIRE].

[12] CMS collaboration, Search for the associated production of the Higgs boson with a top-quark pair, JHEP 09 (2014) 087 [Erratum ibid. 10 (2014) 106] [arXiv:1408.1682] [INSPIRE].

[13] ATLAS collaboration, Search for the b $\bar{b}$ decay of the standard model Higgs boson in associated $(W / Z) H$ production with the ATLAS detector, JHEP 01 (2015) 069 [arXiv: 1409.6212] [INSPIRE].

[14] CMS collaboration, Evidence for the $125 \mathrm{GeV}$ Higgs boson decaying to a pair of $\tau$ leptons, JHEP 05 (2014) 104 [arXiv:1401.5041] [INSPIRE].

[15] ATLAS collaboration, Observation and measurement of Higgs boson decays to $W W^{*}$ with ATLAS at the LHC, ATLAS-CONF-2014-060 (2014).

[16] ATLAS collaboration, Determination of spin and parity of the Higgs boson in the $W W^{*} \rightarrow e \nu \mu \nu$ decay channel with the ATLAS detector, Eur. Phys. J. C 75 (2015) 231 [arXiv: 1503.03643] [INSPIRE].

[17] CMS collaboration, Measurement of the properties of a Higgs boson in the four-lepton final state, Phys. Rev. D 89 (2014) 092007 [arXiv: 1312.5353] [INSPIRE].

[18] CMS collaboration, Study of the mass and spin-parity of the Higgs boson candidate via its decays to Z boson pairs, Phys. Rev. Lett. 110 (2013) 081803 [arXiv:1212.6639] [INSPIRE].

[19] A. Arbey, M. Battaglia, A. Djouadi, F. Mahmoudi and J. Quevillon, Implications of a 125 GeV Higgs for supersymmetric models, Phys. Lett. B 708 (2012) 162 [arXiv: 1112.3028] [INSPIRE].

[20] D. Ghosh, M. Guchait, S. Raychaudhuri and D. Sengupta, How constrained is the cMSSM?, Phys. Rev. D 86 (2012) 055007 [arXiv: 1205.2283] [InSPIRE].

[21] A. Dighe, D. Ghosh, K.M. Patel and S. Raychaudhuri, Testing times for supersymmetry: looking under the lamp post, Int. J. Mod. Phys. A 28 (2013) 1350134 [arXiv:1303.0721] [INSPIRE].

[22] O. Buchmueller et al., The CMSSM and NUHM1 after LHC Run 1, Eur. Phys. J. C 74 (2014) 2922 [arXiv:1312.5250] [inSPIRE]. 
[23] S.P. Martin, A supersymmetry primer, Adv. Ser. Direct. High Energy Phys. 21 (2010) 1 [hep-ph/9709356] [INSPIRE].

[24] M. Drees, R. Godbole and P. Roy, Theory and phenomenology of sparticles: an account of four-dimensional $N=1$ supersymmetry in high energy physics, World Scientific, Hackensack U.S.A. (2004) [INSPIRE].

[25] J.R. Ellis, K. Enqvist, D.V. Nanopoulos and F. Zwirner, Observables in low-energy superstring models, Mod. Phys. Lett. A 1 (1986) 57 [InSPIRE].

[26] R. Barbieri and G.F. Giudice, Upper bounds on supersymmetric particle masses, Nucl. Phys. B 306 (1988) 63 [InSPIRE].

[27] R. Kitano and Y. Nomura, Supersymmetry, naturalness and signatures at the LHC, Phys. Rev. D 73 (2006) 095004 [hep-ph/0602096] [INSPIRE].

[28] T.J. LeCompte and S.P. Martin, Large Hadron Collider reach for supersymmetric models with compressed mass spectra, Phys. Rev. D 84 (2011) 015004 [arXiv:1105.4304] [INSPIRE].

[29] J. Fan, M. Reece and J.T. Ruderman, Stealth supersymmetry, JHEP 11 (2011) 012 [arXiv:1105.5135] [INSPIRE].

[30] Z. Han, A. Katz, M. Son and B. Tweedie, Boosting searches for natural supersymmetry with R-parity violation via gluino cascades, Phys. Rev. D 87 (2013) 075003 [arXiv:1211.4025] [INSPIRE].

[31] J.A. Evans and Y. Kats, LHC coverage of RPV MSSM with light stops, JHEP 04 (2013) 028 [arXiv:1209.0764] [InSPIRE].

[32] J. Berger, M. Perelstein, M. Saelim and P. Tanedo, The same-sign dilepton signature of RPV/MFV SUSY, JHEP 04 (2013) 077 [arXiv: 1302.2146] [INSPIRE].

[33] S. Biswas, D. Ghosh and S. Niyogi, Multi-leptons and top-jets in the hunt for gluinos in R-parity violating supersymmetry, JHEP 06 (2014) 012 [arXiv: 1312.0549] [INSPIRE].

[34] H. Baer et al., Post-LHC7 fine-tuning in the minimal supergravity/CMSSM model with a 125 GeV Higgs boson, Phys. Rev. D 87 (2013) 035017 [arXiv:1210.3019] [INSPIRE].

[35] MSSM Working Group collaboration, A. Djouadi et al., The minimal supersymmetric standard model: group summary report, hep-ph/9901246 [INSPIRE].

[36] C.F. Berger, J.S. Gainer, J.L. Hewett and T.G. Rizzo, Supersymmetry without prejudice, JHEP 02 (2009) 023 [arXiv:0812.0980] [InSPIRE].

[37] A. Arbey, M. Battaglia, A. Djouadi and F. Mahmoudi, An update on the constraints on the phenomenological MSSM from the new LHC Higgs results, Phys. Lett. B 720 (2013) 153 [arXiv: 1211.4004] [INSPIRE].

[38] B. Dumont, J.F. Gunion and S. Kraml, Phenomenological MSSM in view of the $125 \mathrm{GeV}$ Higgs data, Phys. Rev. D 89 (2014) 055018 [arXiv:1312.7027] [InSPIRE].

[39] J. Berger et al., The CP-violating pMSSM at the intensity frontier, arXiv:1309.7653 [INSPIRE].

[40] J. Berger et al., The CP-violating pMSSM, in preparation.

[41] M. Cahill-Rowley, J.L. Hewett, A. Ismail and T.G. Rizzo, Lessons and prospects from the pMSSM after LHC Run I, Phys. Rev. D 91 (2015) 055002 [arXiv:1407.4130] [INSPIRE]. 
[42] H. Baer, V. Barger, D. Mickelson and M. Padeffke-Kirkland, SUSY models under siege: LHC constraints and electroweak fine-tuning, Phys. Rev. D 89 (2014) 115019 [arXiv: 1404.2277] [INSPIRE].

[43] C. Strege et al., Profile likelihood maps of a 15-dimensional MSSM, JHEP 09 (2014) 081 [arXiv: 1405.0622] [INSPIRE].

[44] L. Roszkowski, E.M. Sessolo and A.J. Williams, Prospects for dark matter searches in the pMSSM, JHEP 02 (2015) 014 [arXiv:1411.5214] [INSPIRE].

[45] K.J. de Vries et al., The pMSSM10 after LHC Run 1, Eur. Phys. J. C 75 (2015) 422 [arXiv: 1504.03260] [INSPIRE].

[46] C. Brust, A. Katz, S. Lawrence and R. Sundrum, SUSY, the third generation and the LHC, JHEP 03 (2012) 103 [arXiv:1110.6670] [InSPIRE].

[47] X.-J. Bi, Q.-S. Yan and P.-F. Yin, Probing light stop pairs at the LHC, Phys. Rev. D 85 (2012) 035005 [arXiv:1111.2250] [INSPIRE].

[48] B. He, T. Li and Q. Shafi, Impact of LHC searches on NLSP top squark and gluino mass, JHEP 05 (2012) 148 [arXiv:1112.4461] [INSPIRE].

[49] T. Plehn, M. Spannowsky and M. Takeuchi, Stop searches in 2012, JHEP 08 (2012) 091 [arXiv:1205.2696] [INSPIRE].

[50] D.E. Kaplan, K. Rehermann and D. Stolarski, Searching for direct stop production in hadronic top data at the LHC, JHEP 07 (2012) 119 [arXiv:1205.5816] [INSPIRE].

[51] S. Sekmen et al., Interpreting LHC SUSY searches in the phenomenological MSSM, JHEP 02 (2012) 075 [arXiv:1109.5119] [InSPIRE].

[52] S.S. AbdusSalam and D. Choudhury, Higgs boson discovery versus sparticles prediction: impact on the pMSSM's posterior samples from a Bayesian global fit,

Universal J. Phys. Appl. 2 (2014) 155 [arXiv:1210.3331] [inSPIRE].

[53] M. Chakraborti, U. Chattopadhyay, A. Choudhury, A. Datta and S. Poddar, The electroweak sector of the pMSSM in the light of LHC-8 TeV and other data, JHEP 07 (2014) 019 [arXiv: 1404.4841] [INSPIRE].

[54] A. Fowlie, K. Kowalska, L. Roszkowski, E.M. Sessolo and Y.-L.S. Tsai, Dark matter and collider signatures of the MSSM, Phys. Rev. D 88 (2013) 055012 [arXiv:1306.1567] [INSPIRE].

[55] S. Henrot-Versillé et al., Constraining supersymmetry using the relic density and the Higgs boson, Phys. Rev. D 89 (2014) 055017 [arXiv: 1309.6958] [INSPIRE].

[56] S.S. AbdusSalam, LHC-7 supersymmetry search interpretation within the phenomenological MSSM, Phys. Rev. D 87 (2013) 115012 [arXiv:1211.0999] [INSPIRE].

[57] T. Plehn, M. Spannowsky, M. Takeuchi and D. Zerwas, Stop reconstruction with tagged tops, JHEP 10 (2010) 078 [arXiv: 1006.2833] [INSPIRE].

[58] S. Bornhauser, M. Drees, S. Grab and J.S. Kim, Light stop searches at the LHC in events with two b-jets and missing energy, Phys. Rev. D 83 (2011) 035008 [arXiv:1011.5508] [INSPIRE].

[59] Y. Bai, H.-C. Cheng, J. Gallicchio and J. Gu, Stop the top background of the stop search, JHEP 07 (2012) 110 [arXiv: 1203.4813] [INSPIRE]. 
[60] Z. Han, A. Katz, D. Krohn and M. Reece, (Light) stop signs, JHEP 08 (2012) 083 [arXiv: 1205.5808] [INSPIRE].

[61] M.L. Graesser and J. Shelton, Hunting mixed top squark decays, Phys. Rev. Lett. 111 (2013) 121802 [arXiv:1212.4495] [INSPIRE].

[62] C. Kilic and B. Tweedie, Cornering light stops with dileptonic $m_{T 2}$, JHEP 04 (2013) 110 [arXiv:1211.6106] [INSPIRE].

[63] D. Ghosh and D. Sengupta, Searching the sbottom in the four lepton channel at the LHC, Eur. Phys. J. C 73 (2013) 2342 [arXiv:1209.4310] [INSPIRE].

[64] Y. Bai, H.-C. Cheng, J. Gallicchio and J. Gu, A toolkit of the stop search via the chargino decay, JHEP 08 (2013) 085 [arXiv: 1304.3148] [INSPIRE].

[65] A. Chakraborty, D.K. Ghosh, D. Ghosh and D. Sengupta, Stop and sbottom search using dileptonic $M_{T 2}$ variable and boosted top technique at the LHC, JHEP 10 (2013) 122 [arXiv:1303.5776] [INSPIRE].

[66] B. Dutta et al., Top squark searches using dilepton invariant mass distributions and bino-Higgsino dark matter at the LHC, Phys. Rev. D 87 (2013) 095007 [arXiv: 1302.3231] [INSPIRE].

[67] M.R. Buckley, T. Plehn and M.J. Ramsey-Musolf, Top squark with mass close to the top quark, Phys. Rev. D 90 (2014) 014046 [arXiv:1403.2726] [INSPIRE].

[68] M. Czakon, A. Mitov, M. Papucci, J.T. Ruderman and A. Weiler, Closing the stop gap, Phys. Rev. Lett. 113 (2014) 201803 [arXiv:1407.1043] [INSPIRE].

[69] T. Eifert and B. Nachman, Sneaky light stop, Phys. Lett. B 743 (2015) 218 [arXiv: 1410.7025] [INSPIRE].

[70] W.S. Cho et al., Improving the sensitivity of stop searches with on-shell constrained invariant mass variables, JHEP 05 (2015) 040 [arXiv: 1411.0664] [INSPIRE].

[71] G. Ferretti, R. Franceschini, C. Petersson and R. Torre, Spot the stop with a b-tag, Phys. Rev. Lett. 114 (2015) 201801 [arXiv:1502.01721] [INSPIRE].

[72] K. Rolbiecki and J. Tattersall, Refining light stop exclusion limits with $W^{+} W^{-}$cross sections, arXiv: 1505.05523 [INSPIRE].

[73] B. Nachman and C.G. Lester, Significance variables, Phys. Rev. D 88 (2013) 075013 [arXiv: 1303.7009] [INSPIRE].

[74] K. Krizka, A. Kumar and D.E. Morrissey, Very light scalar top quarks at the LHC, Phys. Rev. D 87 (2013) 095016 [arXiv:1212.4856] [INSPIRE].

[75] M. Drees, M. Hanussek and J.S. Kim, Light stop searches at the LHC with monojet events, Phys. Rev. D 86 (2012) 035024 [arXiv:1201.5714] [inSPIRE].

[76] D.S.M. Alves, M.R. Buckley, P.J. Fox, J.D. Lykken and C.-T. Yu, Stops and $E_{T}$ : the shape of things to come, Phys. Rev. D 87 (2013) 035016 [arXiv:1205.5805] [InSPIRE].

[77] K. Ghosh, K. Huitu, J. Laamanen, L. Leinonen and J. Laamanen, Top quark jets as a probe of the constrained minimal supersymmetric standard model with a degenerate top squark and lightest supersymmetric particle, Phys. Rev. Lett. 110 (2013) 141801 [arXiv: 1207.2429] [INSPIRE].

[78] A. Delgado, G.F. Giudice, G. Isidori, M. Pierini and A. Strumia, The light stop window, Eur. Phys. J. C 73 (2013) 2370 [arXiv:1212.6847] [InSPIRE]. 
[79] D. Ghosh, Boosted dibosons from mixed heavy top squarks, Phys. Rev. D 88 (2013) 115013 [arXiv: 1308.0320] [INSPIRE].

[80] G. Bélanger, D. Ghosh, R. Godbole, M. Guchait and D. Sengupta, Probing the flavor violating scalar top quark signal at the LHC, Phys. Rev. D 89 (2014) 015003 [arXiv: 1308.6484] [INSPIRE].

[81] B. Dutta et al., Probing compressed top squark scenarios at the LHC at $14 \mathrm{TeV}$, Phys. Rev. D 90 (2014) 095022 [arXiv: 1312.1348] [INSPIRE].

[82] R. Gröber, M.M. Mühlleitner, E. Popenda and A. Wlotzka, Light stop decays: implications for LHC searches, Eur. Phys. J. C 75 (2015) 420 [arXiv:1408.4662] [InSPIRE].

[83] R. Gröber, M. Mühlleitner, E. Popenda and A. Wlotzka, Light stop decays into Wb $\tilde{\chi}_{1}^{0}$ near the kinematic threshold, Phys. Lett. B 747 (2015) 144 [arXiv:1502.05935] [InSPIRE].

[84] K.-i. Hikasa, J. Li, L. Wu and J.M. Yang, Mono-stop production as a probe of natural SUSY at the LHC, arXiv:1505.06006 [INSPIRE].

[85] S. Kraml et al., SModelS: a tool for interpreting simplified-model results from the LHC and its application to supersymmetry, Eur. Phys. J. C 74 (2014) 2868 [arXiv:1312.4175] [INSPIRE].

[86] S. Kraml et al., SModelS v1.0: a short user guide, arXiv:1412.1745 [INSPIRE].

[87] A. Djouadi, J.-L. Kneur and G. Moultaka, SuSpect: a Fortran code for the supersymmetric and Higgs particle spectrum in the MSSM, Comput. Phys. Commun. 176 (2007) 426 [hep-ph/0211331] [INSPIRE].

[88] D. Chowdhury, R.M. Godbole, K.A. Mohan and S.K. Vempati, Charge and color breaking constraints in MSSM after the Higgs discovery at LHC, JHEP 02 (2014) 110 [arXiv: 1310.1932] [INSPIRE].

[89] J.E. Camargo-Molina, B. Garbrecht, B. O'Leary, W. Porod and F. Staub, Constraining the Natural MSSM through tunneling to color-breaking vacua at zero and non-zero temperature, Phys. Lett. B 737 (2014) 156 [arXiv:1405.7376] [INSPIRE].

[90] N. Blinov and D.E. Morrissey, Vacuum stability and the MSSM Higgs mass, JHEP 03 (2014) 106 [arXiv:1310.4174] [InSPIRE].

[91] P. Bechtle, O. Brein, S. Heinemeyer, G. Weiglein and K.E. Williams, HiggsBounds: confronting arbitrary Higgs sectors with exclusion bounds from LEP and the Tevatron, Comput. Phys. Commun. 181 (2010) 138 [arXiv:0811.4169] [INSPIRE].

[92] P. Bechtle, O. Brein, S. Heinemeyer, G. Weiglein and K.E. Williams, HiggsBounds 2.0.0: confronting neutral and charged Higgs sector predictions with exclusion bounds from LEP and the Tevatron, Comput. Phys. Commun. 182 (2011) 2605 [arXiv:1102.1898] [InSPIRE].

[93] P. Bechtle, S. Heinemeyer, O. Stål, T. Stefaniak and G. Weiglein, HiggsSignals: confronting arbitrary Higgs sectors with measurements at the Tevatron and the LHC,

Eur. Phys. J. C 74 (2014) 2711 [arXiv:1305.1933] [INSPIRE].

[94] S. Heinemeyer, W. Hollik and G. Weiglein, FeynHiggs: a program for the calculation of the masses of the neutral CP even Higgs bosons in the MSSM, Comput. Phys. Commun. 124 (2000) 76 [hep-ph/9812320] [INSPIRE].

[95] LHCb collaboration, Measurement of form-factor-independent observables in the decay $B^{0} \rightarrow K^{* 0} \mu^{+} \mu^{-}$, Phys. Rev. Lett. 111 (2013) 191801 [arXiv:1308.1707] [INSPIRE]. 
[96] LHCb collaboration, Test of lepton universality using $B^{+} \rightarrow K^{+} \ell^{+} \ell^{-}$decays, Phys. Rev. Lett. 113 (2014) 151601 [arXiv:1406.6482] [InSPIRE].

[97] S. Descotes-Genon, J. Matias and J. Virto, Understanding the $B \rightarrow K^{*} \mu^{+} \mu^{-}$anomaly, Phys. Rev. D 88 (2013) 074002 [arXiv:1307.5683] [INSPIRE].

[98] R. Gauld, F. Goertz and U. Haisch, On minimal $Z^{\prime}$ explanations of the $B \rightarrow K^{*} \mu^{+} \mu^{-}$ anomaly, Phys. Rev. D 89 (2014) 015005 [arXiv: 1308.1959] [INSPIRE].

[99] A. Datta, M. Duraisamy and D. Ghosh, Explaining the $B \rightarrow K^{*} \mu^{+} \mu^{-}$data with scalar interactions, Phys. Rev. D 89 (2014) 071501 [arXiv:1310.1937] [INSPIRE].

[100] W. Altmannshofer, S. Gori, M. Pospelov and I. Yavin, Quark flavor transitions in $L_{\mu}-L_{\tau}$ models, Phys. Rev. D 89 (2014) 095033 [arXiv: 1403.1269] [INSPIRE].

[101] D. Ghosh, M. Nardecchia and S.A. Renner, Hint of lepton flavour non-universality in $B$ meson decays, JHEP 12 (2014) 131 [arXiv:1408.4097] [INSPIRE].

[102] W. Altmannshofer and D.M. Straub, New physics in $b \rightarrow s$ transitions after LHC run 1, Eur. Phys. J. C 75 (2015) 382 [arXiv:1411.3161] [InSPIRE].

[103] F. Mahmoudi, SuperIso v2.3: a program for calculating flavor physics observables in supersymmetry, Comput. Phys. Commun. 180 (2009) 1579 [arXiv:0808.3144] [INSPIRE].

[104] Muon G-2 collaboration, G.W. Bennett et al., Final report of the muon E821 anomalous magnetic moment measurement at BNL, Phys. Rev. D 73 (2006) 072003 [hep-ex/0602035] [INSPIRE].

[105] C. Gnendiger, D. Stöckinger and H. Stöckinger-Kim, The electroweak contributions to $(g-2)_{\mu}$ after the Higgs boson mass measurement, Phys. Rev. D 88 (2013) 053005 [arXiv: 1306.5546] [INSPIRE].

[106] B. Bhattacherjee, A. Dighe, D. Ghosh and S. Raychaudhuri, Do new data on $B^{+} \rightarrow \tau^{+} \nu_{\tau}$ decays point to an early discovery of supersymmetry at the LHC?,

Phys. Rev. D 83 (2011) 094026 [arXiv: 1012.1052] [INSPIRE].

[107] BeLLE collaboration, I. Adachi et al., Evidence for $B^{-} \rightarrow \tau^{-} \bar{\nu}_{\tau}$ with a hadronic tagging method using the full data sample of Belle, Phys. Rev. Lett. 110 (2013) 131801 [arXiv:1208.4678] [INSPIRE].

[108] BABAR collaboration, J.P. Lees et al., Evidence for an excess of $\bar{B} \rightarrow D^{(*)} \tau^{-} \bar{\nu}_{\tau}$ decays, Phys. Rev. Lett. 109 (2012) 101802 [arXiv:1205.5442] [INSPIRE].

[109] S. Fajfer, J.F. Kamenik, I. Nisandzic and J. Zupan, Implications of lepton flavor universality violations in B decays, Phys. Rev. Lett. 109 (2012) 161801 [arXiv:1206.1872] [INSPIRE].

[110] S. Fajfer, J.F. Kamenik and I. Nisandzic, On the $B \rightarrow D^{*} \tau \bar{\nu}_{\tau}$ sensitivity to new physics, Phys. Rev. D 85 (2012) 094025 [arXiv:1203.2654] [INSPIRE].

[111] A. Datta, M. Duraisamy and D. Ghosh, Diagnosing new physics in $b \rightarrow c \tau \nu_{\tau}$ decays in the light of the recent BaBar result, Phys. Rev. D 86 (2012) 034027 [arXiv:1206.3760] [INSPIRE].

[112] Planck collaboration, P.A.R. Ade et al., Planck 2013 results. XVI. Cosmological parameters, Astron. Astrophys. 571 (2014) A16 [arXiv:1303.5076] [INSPIRE].

[113] N. Baro, F. Boudjema and A. Semenov, Full one-loop corrections to the relic density in the MSSM: a few examples, Phys. Lett. B 660 (2008) 550 [arXiv:0710.1821] [INSPIRE]. 
[114] N. Baro, F. Boudjema, G. Chalons and S. Hao, Relic density at one-loop with gauge boson pair production, Phys. Rev. D 81 (2010) 015005 [arXiv:0910.3293] [INSPIRE].

[115] F. Boudjema, G. Drieu La Rochelle and S. Kulkarni, One-loop corrections, uncertainties and approximations in neutralino annihilations: examples, Phys. Rev. D 84 (2011) 116001 [arXiv:1108.4291] [INSPIRE].

[116] J. Harz, B. Herrmann, M. Klasen, K. Kovarik and Q.L. Boulc'h, Neutralino-stop coannihilation into electroweak gauge and Higgs bosons at one loop, Phys. Rev. D 87 (2013) 054031 [arXiv:1212.5241] [INSPIRE].

[117] J. Harz, B. Herrmann, M. Klasen and K. Kovarik, One-loop corrections to neutralino-stop coannihilation revisited, Phys. Rev. D 91 (2015) 034028 [arXiv: 1409.2898] [INSPIRE].

[118] Planck collaboration, P.A.R. Ade et al., Planck 2015 results. XIII. Cosmological parameters, arXiv:1502.01589 [INSPIRE].

[119] LUX collaboration, D.S. Akerib et al., First results from the LUX dark matter experiment at the Sanford Underground Research Facility, Phys. Rev. Lett. 112 (2014) 091303 [arXiv: 1310.8214] [INSPIRE].

[120] Fermi-LAT collaboration, M. Ackermann et al., Searching for dark matter annihilation from Milky Way dwarf spheroidal galaxies with six years of Fermi-LAT data, arXiv: 1503.02641 [INSPIRE].

[121] PAMELA collaboration, O. Adriani et al., PAMELA results on the cosmic-ray antiproton flux from $60 \mathrm{MeV}$ to $180 \mathrm{GeV}$ in kinetic energy, Phys. Rev. Lett. 105 (2010) 121101 [arXiv: 1007.0821] [INSPIRE].

[122] A. Kounine, Latest results from the Alpha Magnetic Spectrometer: positron fraction and antiproton/proton ratio, talk presented at the AMS Days at CERN, Geneva Switzerland, 15-17 Apr 2015, https://indico.cern.ch/event/381134/contribution/3/attachments/ 759339/1041605/AMS_positrons_antiprotons.pdf.

[123] PAMELA collaboration, O. Adriani et al., An anomalous positron abundance in cosmic rays with energies 1.5-100 GeV, Nature 458 (2009) 607 [arXiv:0810.4995] [INSPIRE].

[124] AMS collaboration, M. Aguilar et al., First result from the Alpha Magnetic Spectrometer on the International Space Station: precision measurement of the positron fraction in primary cosmic rays of 0.5-350 GeV, Phys. Rev. Lett. 110 (2013) 141102 [INSPIRE].

[125] G. Bélanger, F. Boudjema, A. Pukhov and A. Semenov, MicrOMEGAs_3: a program for calculating dark matter observables, Comput. Phys. Commun. 185 (2014) 960 [arXiv: 1305.0237] [INSPIRE].

[126] OPAL collaboration, G. Abbiendi et al., Search for chargino and neutralino production at $\sqrt{s}=192 \mathrm{GeV}$ to $209 \mathrm{GeV}$ at LEP, Eur. Phys. J. C 35 (2004) 1 [hep-ex/0401026] [INSPIRE].

[127] P.Z. Skands et al., SUSY Les Houches accord: interfacing SUSY spectrum calculators, decay packages and event generators, JHEP 07 (2004) 036 [hep-ph/0311123] [INSPIRE].

[128] J. Alwall et al., A standard format for Les Houches event files, Comput. Phys. Commun. 176 (2007) 300 [hep-ph/0609017] [INSPIRE].

[129] Extending the SLHA: cross section information, http://phystev.in2p3.fr/wiki/2013:groups:tools:slha. 
[130] T. Sjöstrand, S. Mrenna and P.Z. Skands, PYTHIA 6.4 physics and manual, JHEP 05 (2006) 026 [hep-ph/0603175] [INSPIRE].

[131] http://pauli.uni-muenster.de/ãkule_01/nllwiki/index.php/NLL-fast.

[132] W. Beenakker, R. Hopker, M. Spira and P.M. Zerwas, Squark and gluino production at hadron colliders, Nucl. Phys. B 492 (1997) 51 [hep-ph/9610490] [InSPIRE].

[133] A. Kulesza and L. Motyka, Threshold resummation for squark-antisquark and gluino-pair production at the LHC, Phys. Rev. Lett. 102 (2009) 111802 [arXiv:0807.2405] [INSPIRE].

[134] A. Kulesza and L. Motyka, Soft gluon resummation for the production of gluino-gluino and squark-antisquark pairs at the LHC, Phys. Rev. D 80 (2009) 095004 [arXiv:0905.4749] [INSPIRE].

[135] W. Beenakker et al., Soft-gluon resummation for squark and gluino hadroproduction, JHEP 12 (2009) 041 [arXiv:0909.4418] [INSPIRE].

[136] W. Beenakker et al., Squark and gluino hadroproduction, Int. J. Mod. Phys. A 26 (2011) 2637 [arXiv:1105.1110] [inSPIRE].

[137] W. Beenakker, M. Krämer, T. Plehn, M. Spira and P.M. Zerwas, Stop production at hadron colliders, Nucl. Phys. B 515 (1998) 3 [hep-ph/9710451] [INSPIRE].

[138] W. Beenakker et al., Supersymmetric top and bottom squark production at hadron colliders, JHEP 08 (2010) 098 [arXiv: 1006.4771] [INSPIRE].

[139] ATLAS collaboration, Search for new phenomena in final states with large jet multiplicities and missing transverse momentum at $\sqrt{s}=8 \mathrm{TeV}$ proton-proton collisions using the ATLAS experiment, JHEP 10 (2013) 130 [Erratum ibid. 01 (2014) 109] [arXiv:1308.1841] [INSPIRE].

[140] ATLAS collaboration, Search for direct third-generation squark pair production in final states with missing transverse momentum and two b-jets in $\sqrt{s}=8 \mathrm{TeV}$ pp collisions with the ATLAS detector, JHEP 10 (2013) 189 [arXiv:1308.2631] [INSPIRE].

[141] ATLAS collaboration, Search for direct production of charginos and neutralinos in events with three leptons and missing transverse momentum in $\sqrt{s}=8 \mathrm{TeV}$ pp collisions with the ATLAS detector, JHEP 04 (2014) 169 [arXiv: 1402.7029] [INSPIRE].

[142] ATLAS collaboration, Search for direct top-squark pair production in final states with two leptons in pp collisions at $\sqrt{s}=8 \mathrm{TeV}$ with the ATLAS detector, JHEP 06 (2014) 124 [arXiv: 1403.4853] [INSPIRE].

[143] ATLAS collaboration, Search for direct production of charginos, neutralinos and sleptons in final states with two leptons and missing transverse momentum in pp collisions at $\sqrt{s}=8 \mathrm{TeV}$ with the ATLAS detector, JHEP 05 (2014) 071 [arXiv:1403.5294] [INSPIRE].

[144] ATLAS collaboration, Search for squarks and gluinos with the ATLAS detector in final states with jets and missing transverse momentum using $\sqrt{s}=8$ TeV proton-proton collision data, JHEP 09 (2014) 176 [arXiv:1405.7875] [INSPIRE].

[145] ATLAS collaboration, Search for the direct production of charginos, neutralinos and staus in final states with at least two hadronically decaying taus and missing transverse momentum in pp collisions at $\sqrt{s}=8 \mathrm{TeV}$ with the ATLAS detector, JHEP 10 (2014) 096 [arXiv: 1407.0350] [INSPIRE]. 
[146] ATLAS collaboration, Search for top squark pair production in final states with one isolated lepton, jets and missing transverse momentum in $\sqrt{s}=8 \mathrm{TeV}$ pp collisions with the ATLAS detector, JHEP 11 (2014) 118 [arXiv:1407.0583] [INSPIRE].

[147] ATLAS collaboration, Search for supersymmetry in final states with two same-sign leptons, jets and missing transverse momentum with the ATLAS detector in pp collisions at $\sqrt{s}=8 \mathrm{TeV}$, ATLAS-CONF-2012-105 (2012).

[148] ATLAS collaboration, Search for strongly produced superpartners in final states with two same sign leptons with the ATLAS detector using $21 \mathrm{fb}^{-1}$ of proton-proton collisions at $\sqrt{s}=8 \mathrm{TeV}$, ATLAS-CONF-2013-007 (2013).

[149] ATLAS collaboration, Search for direct production of the top squark in the all-hadronic $t \bar{t}+E_{T}^{\text {miss }}$ final state in $21 \mathrm{fb}^{-1}$ of p-pcollisions at $\sqrt{s}=8 \mathrm{TeV}$ with the ATLAS detector, ATLAS-CONF-2013-024 (2013).

[150] ATLAS collaboration, Search for strong production of supersymmetric particles in final states with missing transverse momentum and at least three b-jets using $20.1 \mathrm{fb}^{-1}$ of $p p$ collisions at $\sqrt{s}=8 \mathrm{TeV}$ with the ATLAS detector, ATLAS-CONF-2013-061 (2013).

[151] ATLAS collaboration, Searches for direct scalar top pair production in final states with two leptons using the stransverse mass variable and a multivariate analysis technique in $\sqrt{s}=8 \mathrm{TeV}$ pp collisions using 20.3 $\mathrm{fb}^{-1}$ of ATLAS data, ATLAS-CONF-2013-065 (2013).

[152] CMS collaboration, Search for gluino mediated bottom- and top-squark production in multijet final states in pp collisions at 8 TeV, Phys. Lett. B 725 (2013) 243 [arXiv:1305.2390] [INSPIRE].

[153] CMS collaboration, Search for supersymmetry in hadronic final states with missing transverse energy using the variables $\alpha_{T}$ and b-quark multiplicity in pp collisions at $\sqrt{s}=8 \mathrm{TeV}$, Eur. Phys. J. C 73 (2013) 2568 [arXiv:1303.2985] [INSPIRE].

[154] CMS collaboration, Search for anomalous production of events with three or more leptons in pp collisions at $\sqrt{s}=8 \mathrm{TeV}$, Phys. Rev. D 90 (2014) 032006 [arXiv:1404.5801] [InSPIRE].

[155] CMS collaboration, Searches for electroweak production of charginos, neutralinos and sleptons decaying to leptons and $W, Z$ and Higgs bosons in pp collisions at $8 \mathrm{TeV}$, Eur. Phys. J. C 74 (2014) 3036 [arXiv: 1405.7570] [inSPIRE].

[156] CMS collaboration, Search for supersymmetry in pp collisions at $\sqrt{s}=8 \mathrm{TeV}$ in events with a single lepton, large jet multiplicity and multiple b jets, Phys. Lett. B 733 (2014) 328 [arXiv:1311.4937] [INSPIRE].

[157] CMS collaboration, Search for top-squark pair production in the single-lepton final state in pp collisions at $\sqrt{s}=8 \mathrm{TeV}$, Eur. Phys. J. C 73 (2013) 2677 [arXiv:1308.1586] [INSPIRE].

[158] CMS collaboration, Search for new physics in the multijet and missing transverse momentum final state in proton-proton collisions at $\sqrt{s}=8 \mathrm{TeV}$, JHEP 06 (2014) 055 [arXiv: 1402.4770] [INSPIRE].

[159] CMS collaboration, Search for new physics in events with same-sign dileptons and jets in pp collisions at $\sqrt{s}=8 \mathrm{TeV}$, JHEP 01 (2014) 163 [Erratum ibid. 01 (2015) 014] [arXiv:1311.6736] [INSPIRE].

[160] CMS collaboration, Search for supersymmetry in pp collisions at $\sqrt{s}=8 \mathrm{TeV}$ in events with three leptons and at least one b-tagged jet, CMS-PAS-SUS-13-008 (2013). 
[161] CMS collaboration, Search for supersymmetry in pp collisions at $\sqrt{s}=8$ Tev in events with two opposite sign leptons, large number of jets, b-tagged jets, and large missing transverse energy, CMS-PAS-SUS-13-016 (2013).

[162] CMS collaboration, Search for direct production of bottom squark pairs, CMS-PAS-SUS-13-018 (2014).

[163] CMS collaboration, Search for supersymmetry in hadronic final states using $M_{T 2}$ with the CMS detector at $\sqrt{s}=8 \mathrm{TeV}$, CMS-PAS-SUS-13-019 (2014).

[164] CMS collaboration, Exclusion limits on gluino and top-squark pair production in natural SUSY scenarios with inclusive razor and exclusive single-lepton searches at $8 \mathrm{TeV}$, CMS-PAS-SUS-14-011 (2014).

[165] ATLAS collaboration, Search for pair-produced third-generation squarks decaying via charm quarks or in compressed supersymmetric scenarios in pp collisions at $\sqrt{s}=8 \mathrm{TeV}$ with the ATLAS detector, Phys. Rev. D 90 (2014) 052008 [arXiv:1407.0608] [InSPIRE].

[166] CMS collaboration, Search for top squarks decaying to a charm quark and a neutralino in events with a jet and missing transverse momentum, CMS-PAS-SUS-13-009 (2014).

[167] J.S. Kim, D. Schmeier, J. Tattersall and K. Rolbiecki, A framework to create customised LHC analyses within CheckMATE, arXiv:1503.01123 [INSPIRE].

[168] B. Dumont et al., Toward a public analysis database for LHC new physics searches using MADANALYSIS 5, Eur. Phys. J. C 75 (2015) 56 [arXiv:1407.3278] [inSPIRE].

[169] E. Conte, B. Dumont, B. Fuks and C. Wymant, Designing and recasting LHC analyses with MadAnalysis 5, Eur. Phys. J. C 74 (2014) 3103 [arXiv:1405.3982] [INSPIRE].

[170] CMS collaboration, Search for top-squark pairs decaying into Higgs or $Z$ bosons in pp collisions at $\sqrt{s}=8 \mathrm{TeV}$, Phys. Lett. B 736 (2014) 371 [arXiv:1405.3886] [INSPIRE].

[171] ATLAS collaboration, Search for direct pair production of the top squark in all-hadronic final states in proton-proton collisions at $\sqrt{s}=8 \mathrm{TeV}$ with the ATLAS detector, JHEP 09 (2014) 015 [arXiv: 1406.1122] [INSPIRE].

[172] CMS collaboration, Search for supersymmetry using razor variables in events with b-tagged jets in pp collisions at $\sqrt{s}=8 \mathrm{TeV}$, Phys. Rev. D 91 (2015) 052018 [arXiv:1502.00300] [INSPIRE].

[173] ATLAS collaboration, Search for new particles in events with one lepton and missing transverse momentum in pp collisions at $\sqrt{s}=8 \mathrm{TeV}$ with the ATLAS detector, JHEP 09 (2014) 037 [arXiv: 1407.7494] [INSPIRE].

[174] CMS collaboration, Search for physics beyond the standard model in final states with a lepton and missing transverse energy in proton-proton collisions at $\sqrt{s}=8 \mathrm{TeV}$, Phys. Rev. D 91 (2015) 092005 [arXiv:1408.2745] [INSPIRE].

[175] ATLAS collaboration, Search for new phenomena in events with a photon and missing transverse momentum in pp collisions at $\sqrt{s}=8 \mathrm{TeV}$ with the ATLAS detector, Phys. Rev. D 91 (2015) 012008 [arXiv:1411.1559] [INSPIRE].

[176] J. Alwall et al., The automated computation of tree-level and next-to-leading order differential cross sections and their matching to parton shower simulations, JHEP 07 (2014) 079 [arXiv:1405.0301] [InSPIRE]. 
[177] A. Djouadi, J.L. Kneur and G. Moultaka, Associated production of Higgs bosons with scalar quarks at future hadron and $e^{+} e^{-}$colliders, Nucl. Phys. B 569 (2000) 53 [hep-ph/9903218] [INSPIRE].

[178] G. Bélanger, F. Boudjema and K. Sridhar, SUSY Higgs at the LHC: large stop mixing effects and associated production, Nucl. Phys. B 568 (2000) 3 [hep-ph/9904348] [INSPIRE].

[179] A. Djouadi, L. Maiani, A. Polosa, J. Quevillon and V. Riquer, Fully covering the MSSM Higgs sector at the LHC, JHEP 06 (2015) 168 [arXiv: 1502.05653] [INSPIRE].

[180] R.V. Harlander and W.B. Kilgore, Next-to-next-to-leading order Higgs production at hadron colliders, Phys. Rev. Lett. 88 (2002) 201801 [hep-ph/0201206] [INSPIRE].

[181] R.V. Harlander and W.B. Kilgore, Higgs boson production in bottom quark fusion at next-to-next-to leading order, Phys. Rev. D 68 (2003) 013001 [hep-ph/0304035] [INSPIRE].

[182] U. Aglietti, R. Bonciani, G. Degrassi and A. Vicini, Two loop light fermion contribution to Higgs production and decays, Phys. Lett. B 595 (2004) 432 [hep-ph/0404071] [INSPIRE].

[183] R. Harlander and P. Kant, Higgs production and decay: analytic results at next-to-leading order QCD, JHEP 12 (2005) 015 [hep-ph/0509189] [INSPIRE].

[184] R. Bonciani, G. Degrassi and A. Vicini, On the generalized harmonic polylogarithms of one complex variable, Comput. Phys. Commun. 182 (2011) 1253 [arXiv:1007.1891] [InSPIRE].

[185] G. Degrassi and P. Slavich, NLO QCD bottom corrections to Higgs boson production in the MSSM, JHEP 11 (2010) 044 [arXiv: 1007.3465] [INSPIRE].

[186] G. Degrassi, S. Di Vita and P. Slavich, NLO QCD corrections to pseudoscalar Higgs production in the MSSM, JHEP 08 (2011) 128 [arXiv:1107.0914] [INSPIRE].

[187] G. Degrassi, S. Di Vita and P. Slavich, On the NLO QCD corrections to the production of the heaviest neutral Higgs scalar in the MSSM, Eur. Phys. J. C 72 (2012) 2032 [arXiv: 1204.1016] [INSPIRE].

[188] R.V. Harlander, S. Liebler and H. Mantler, SusHi: a program for the calculation of Higgs production in gluon fusion and bottom-quark annihilation in the standard model and the MSSM, Comput. Phys. Commun. 184 (2013) 1605 [arXiv: 1212.3249] [InSPIRE].

[189] A. Arbey, M. Battaglia and F. Mahmoudi, Supersymmetric heavy Higgs bosons at the LHC, Phys. Rev. D 88 (2013) 015007 [arXiv: 1303.7450] [INSPIRE].

[190] B. Bhattacherjee, A. Chakraborty and A. Choudhury, Status of MSSM Higgs sector using global analysis and direct search bounds and future prospects at the $H L-L H C$, arXiv: 1504.04308 [INSPIRE].

[191] CMS collaboration, Search for neutral MSSM Higgs bosons decaying to a pair of tau leptons in pp collisions, JHEP 10 (2014) 160 [arXiv:1408.3316] [INSPIRE].

[192] K.J.F. Gaemers and F. Hoogeveen, Higgs production and decay into heavy flavors with the gluon fusion mechanism, Phys. Lett. B 146 (1984) 347 [INSPIRE].

[193] D. Dicus, A. Stange and S. Willenbrock, Higgs decay to top quarks at hadron colliders, Phys. Lett. B 333 (1994) 126 [hep-ph/9404359] [INSPIRE].

[194] W. Bernreuther, M. Flesch and P. Haberl, Signatures of Higgs bosons in the top quark decay channel at hadron colliders, Phys. Rev. D 58 (1998) 114031 [hep-ph/9709284] [INSPIRE].

[195] R. Frederix and F. Maltoni, Top pair invariant mass distribution: a window on new physics, JHEP 01 (2009) 047 [arXiv:0712.2355] [INSPIRE]. 


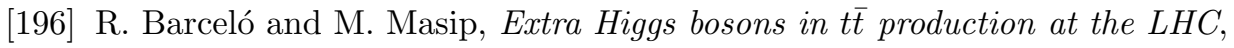
Phys. Rev. D 81 (2010) 075019 [arXiv:1001.5456] [INSPIRE].

[197] S. Moretti and D.A. Ross, On the top-antitop invariant mass spectrum at the LHC from a Higgs boson signal perspective, Phys. Lett. B 712 (2012) 245 [arXiv:1203.3746] [INSPIRE].

[198] N. Craig, F. D'Eramo, P. Draper, S. Thomas and H. Zhang, The hunt for the rest of the Higgs bosons, JHEP 06 (2015) 137 [arXiv: 1504.04630] [INSPIRE].

[199] V. Barger, T. Han and D.G.E. Walker, Top quark pairs at high invariant mass: a model-independent discriminator of new physics at the LHC, Phys. Rev. Lett. 100 (2008) 031801 [hep-ph/0612016] [INSPIRE].

[200] J. Hajer, Y.-Y. Li, T. Liu and J.F.H. Shiu, Heavy Higgs bosons at $14 \mathrm{TeV}$ and $100 \mathrm{TeV}$, arXiv: 1504.07617 [INSPIRE].

[201] M. Bisset et al., Four-lepton LHC events from MSSM Higgs boson decays into neutralino and chargino pairs, JHEP 08 (2009) 037 [arXiv: 0709.1029] [INSPIRE].

[202] R.M. Godbole, M. Guchait, K. Mazumdar, S. Moretti and D.P. Roy, Search for 'invisible' Higgs signals at LHC via associated production with gauge bosons,

Phys. Lett. B 571 (2003) 184 [hep-ph/0304137] [INSPIRE].

[203] O.J.P. Eboli and D. Zeppenfeld, Observing an invisible Higgs boson, Phys. Lett. B 495 (2000) 147 [hep-ph/0009158] [INSPIRE].

[204] CMS collaboration, Search for invisible decays of Higgs bosons in the vector boson fusion and associated ZH production modes, Eur. Phys. J. C 74 (2014) 2980 [arXiv:1404.1344] [INSPIRE].

[205] ATLAS collaboration, Search for invisible decays of a Higgs boson produced in association with a Z boson in ATLAS, Phys. Rev. Lett. 112 (2014) 201802 [arXiv:1402.3244] [INSPIRE].

[206] ATLAS collaboration, Search for invisible decays of the Higgs boson produced in association with a hadronically decaying vector boson in pp collisions at $\sqrt{s}=8 \mathrm{TeV}$ with the ATLAS detector, Eur. Phys. J. C 75 (2015) 337 [arXiv: 1504.04324] [inSPIRE].

[207] D. Ghosh, R. Godbole, M. Guchait, K. Mohan and D. Sengupta, Looking for an invisible Higgs signal at the LHC, Phys. Lett. B 725 (2013) 344 [arXiv:1211.7015] [INSPIRE].

[208] A. Djouadi, A. Falkowski, Y. Mambrini and J. Quevillon, Direct detection of Higgs-portal dark matter at the LHC, Eur. Phys. J. C 73 (2013) 2455 [arXiv:1205.3169] [INSPIRE].

[209] P.S. Bhupal Dev, A. Djouadi, R.M. Godbole, M.M. Muhlleitner and S.D. Rindani, Determining the CP properties of the Higgs boson, Phys. Rev. Lett. 100 (2008) 051801 [arXiv: 0707.2878] [INSPIRE].

[210] F. Boudjema, R.M. Godbole, D. Guadagnoli and K.A. Mohan, Lab-frame observables for probing the top-Higgs interaction, Phys. Rev. D 92 (2015) 015019 [arXiv:1501.03157] [INSPIRE].

[211] T. Moroi and L. Randall, Wino cold dark matter from anomaly mediated SUSY breaking, Nucl. Phys. B 570 (2000) 455 [hep-ph/9906527] [INSPIRE].

[212] L.J. Hall, K. Jedamzik, J. March-Russell and S.M. West, Freeze-in production of FIMP dark matter, JHEP 03 (2010) 080 [arXiv:0911.1120] [INSPIRE]. 
[213] G. Arcadi and P. Ullio, Accurate estimate of the relic density and the kinetic decoupling in non-thermal dark matter models, Phys. Rev. D 84 (2011) 043520 [arXiv:1104.3591] [INSPIRE].

[214] A.J. Williams, C. Boehm, S.M. West and D.A. Vasquez, Regenerating WIMPs in the light of direct and indirect detection, Phys. Rev. D 86 (2012) 055018 [arXiv: 1204.3727] [INSPIRE].

[215] T. Cohen, M. Lisanti, A. Pierce and T.R. Slatyer, Wino dark matter under siege, JCAP 10 (2013) 061 [arXiv: 1307.4082] [INSPIRE].

[216] G. Bélanger, C. Boehm, M. Cirelli, J. Da Silva and A. Pukhov, PAMELA and FERMI-LAT limits on the neutralino-chargino mass degeneracy, JCAP 11 (2012) 028 [arXiv: 1208.5009] [INSPIRE].

[217] J. Fan and M. Reece, In wino veritas? Indirect searches shed light on neutralino dark matter, JHEP 10 (2013) 124 [arXiv:1307.4400] [INSPIRE].

[218] G. Bélanger, J. Da Silva, U. Laa and A. Pukhov, Probing U(1) extensions of the MSSM at the LHC Run I and in dark matter searches, arXiv:1505.06243 [INSPIRE]. 